1 Cryo-EM structure of MukBEF reveals

2 DNA loop entrapment at chromosomal

3 unloading sites

4

5 Frank Bürmann ${ }^{1 *}$, Louise F.H. Funke ${ }^{2}$, Jason W. Chin², Jan Löwe ${ }^{1^{*}}$

\title{
6 Affiliations
}

$7 \quad{ }^{1}$ MRC Laboratory of Molecular Biology, Structural Studies Division, Francis Crick Avenue,

8 Cambridge, UK.

$9 \quad{ }^{2}$ MRC Laboratory of Molecular Biology, Protein and Nucleic Acid Chemistry Division, Francis

10 Crick Avenue, Cambridge, UK.

$11{ }^{*}$ Corresponding author. Email: fburmann@mrc-Imb.cam.ac.uk, jyl@mrc-Imb.cam.ac.uk

12 Keywords

13 SMC, MukBEF, chromosome organization, loop extrusion, cohesin, condensin, cryo-EM

14 Highlights

- Complete atomic structures of the bacterial SMC complex MukBEF on and off DNA.

- MukBEF entraps two DNA double helices when bound to the unloader MatP.

- In vivo topology of DNA loop entrapment determined by cysteine cross-linking.

- Arms of the DNA loop thread through separate compartments of MukBEF. 
bioRxiv preprint doi: https://doi.org/10.1101/2021.06.29.450292; this version posted June 29, 2021. The copyright holder for this preprint

(which was not certified by peer review) is the author/funder, who has granted bioRxiv a license to display the preprint in perpetuity. It is made available under aCC-BY-NC-ND 4.0 International license.

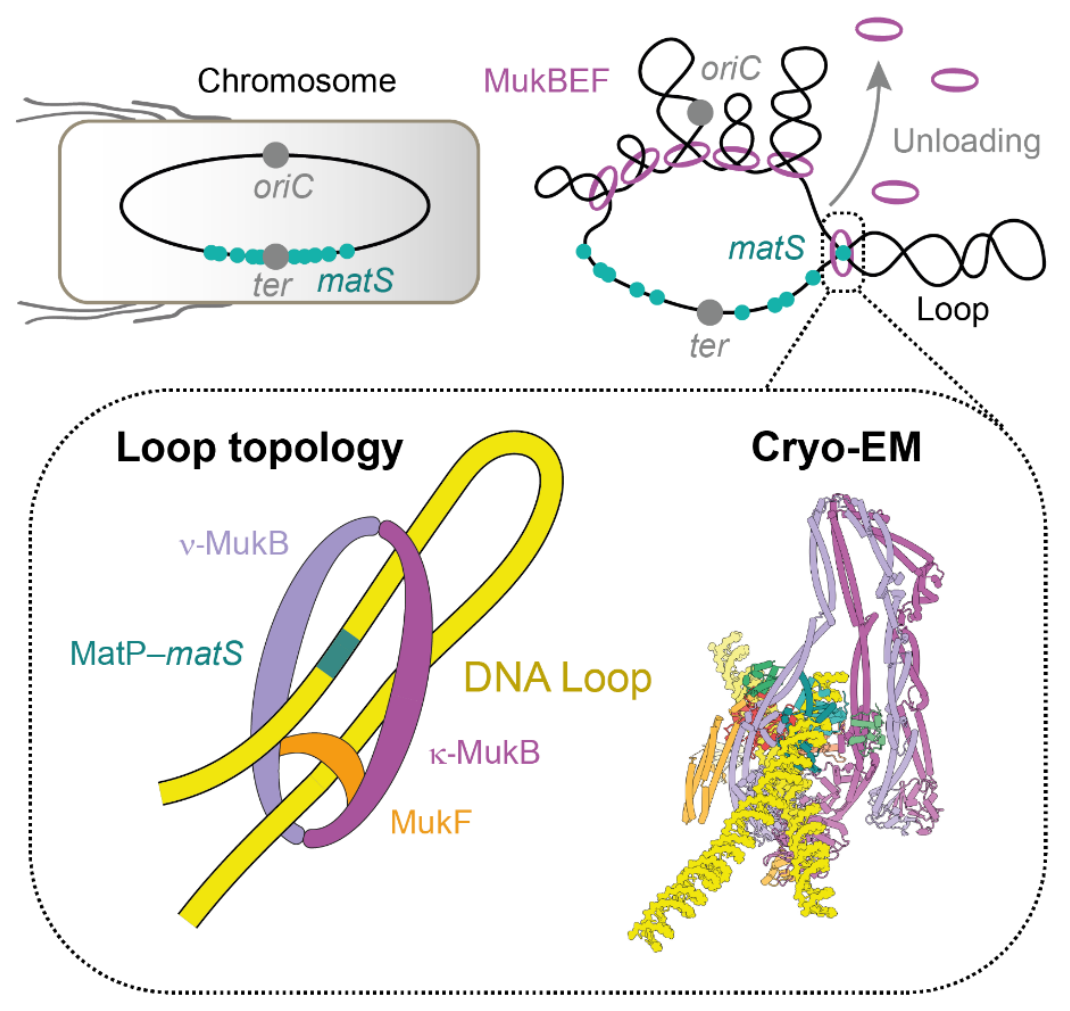




\section{Summary}

24 The ring-like structural maintenance of chromosomes (SMC) complex MukBEF folds the genome of Escherichia coli and related bacteria into large loops, presumably by active DNA loop extrusion. MukBEF activity within the replication terminus macrodomain is suppressed

27 by the sequence specific unloader MatP. Here we present the complete atomic structure of MukBEF in complex with MatP and DNA as determined by electron cryomicroscopy (cryoEM). The complex binds two distinct DNA double helices corresponding to the arms of a plectonemic loop. MatP-bound DNA threads through the MukBEF ring, while the second DNA cross-linking confirms this topology of DNA loop entrapment in vivo. Our findings illuminate how a class of near-ubiquitous DNA organizers with important roles in genome maintenance interacts with the bacterial chromosome.

Associations between molecules due to their topology are known as mechanical bonds

37 (Stoddart, 2009). In eukaryotes as well as prokaryotes, ring-like SMC complexes are thought to structure chromosomes via mechanical bonds with DNA (also referred to as 'DNA entrapment'), and active DNA loop extrusion (Davidson and Peters, 2021; Hassler et al., 2018; Mäkelä and Sherratt, 2020a; Nasmyth, 2001; Yatskevich et al., 2019). These activities have been suggested to enable or facilitate processes such as lengthwise condensation of chromosomes, sister chromatid cohesion, regulation of interactions between enhancers and distant promoters, disentangling of replicated DNA by topoisomerases, DNA recombination, and DNA double-strand break repair. Support for DNA entrapment, whereby the SMC 
complex fully encircles the nucleic acid polymer, comes from experiments probing DNA association after high salt treatment or, more stringently, chemical circularization and denaturation of the complex (Cuylen et al., 2011; Haering et al., 2008; Ivanov and Nasmyth, 2005; Kanno et al., 2015; Murayama and UhImann, 2013; Niki and Yano, 2016; Wilhelm et al., 2015). How DNA entrapment is achieved on the molecular level is less well understood, as SMC complexes contain multiple topological compartments which can or could accommodate one or more DNA double strands (Chapard et al., 2019; Collier et al., 2020; Higashi et al., 2020; Shi et al., 2020; Vazquez Nunez et al., 2019).

At the core of SMC complexes, such as cohesin, condensin, Smc5-6, Smc-ScpAB, and MukBEF, is a tripartite ring composed of two SMC proteins and a kleisin. SMC proteins contain a 50nm-long intra-molecular anti-parallel coiled-coil 'arm', which can fold over at an 'elbow' in MukBEF, cohesin and condensin (Bürmann et al., 2019; Lee et al., 2020; Niki et al., 1992). The arm separates a 'hinge' dimerization domain from an ABC-type ATPase 'head' domain, which undergoes cycles of ATP-dependent dimerization (called 'engagement'), ATP hydrolysis, and disengagement (Hopfner et al., 2000). The kleisin bridges hinge-dimerized SMC proteins in an asymmetric arrangement, whereby its $\mathrm{N}$ - and C-terminal domain bind the SMC 'neck' and 'cap' surfaces, respectively (Bürmann et al., 2013; Gligoris et al., 2014; Haering and Gruber, very head-proximal region of the arm, whereas the cap is part of the head domain. The neckSMC (kappa for cap). 
MukBEF is the SMC complex of $E$. coli and other enterobacteria. Its SMC subunit is MukB, which associates with the kleisin MukF (Woo et al., 2009; Zawadzka et al., 2018). MukF binds the dimeric KITE protein MukE, which is structurally related to ScpB of prokaryotic Smc-ScpAB and Nse1-3 of the Smc5-6 complex (Palecek and Gruber, 2015). MukB ${ }_{2} E_{2} F$ assemblies (hereafter 'MukBEF monomers') dimerize via MukF (Fennell-Fezzie et al., 2005; Woo et al., 2009) into $\mathrm{MukB}_{4} \mathrm{E}_{4} \mathrm{~F}_{2}$ complexes (also called 'MukB dimers of dimers', hereafter simply

'MukBEF dimers'), which are the functional form (Badrinarayanan et al., 2012; Rajasekar et al., 2019).

MukBEF organizes large fractions of the $E$. coli chromosome and is essential for chromosome segregation and cell survival under conditions of fast growth (Danilova et al., 2007; Hiraga et al., 1989; Lioy et al., 2018). In the replication terminus macrodomain (Ter), MukBEF activity is suppressed by unloading at matS sites, which are the signature sequences of Ter (Lioy et al., 2018; Mäkelä and Sherratt, 2020b; Mercier et al., 2008; Nolivos et al., 2016). Unloading of MukBEF drastically changes the loop-size distribution of Ter compared to other chromosomal macrodomains, a process which depends on the matS-binding protein MatP. Although MukBEF function relies on the full ATPase cycle (Badrinarayanan et al., 2012; Woo et al., 2009), association with matS requires ATP-dependent head engagement only (Nolivos et al., 2016). Head engagement and ATP hydrolysis have been suggested to mediate unloading of cohesin in eukaryotes, which involves dissociation of the neck/kleisin interface (Chan et al., 2012; Muir et al., 2020; Murayama and Uhlmann, 2015). This interface also disengages during the ATPase cycle of condensin, raising the possibility that SMC complexes may use related mechanisms for DNA unloading (Hassler et al., 2019). 
92 Here, using cryo-EM single particle analysis we discovered that MukBEF entraps two distinct

93 DNA double helices when bound to the unloader MatP. The DNAs reside in separate

94 compartments, which are located inside the large circumference of the tripartite ring and in

95 a much smaller clamp at the ATPase heads, respectively. Topological mapping by chemical

96 circularization of endogenous MukBEF in cells suggests that these compartments enclose

97 each arm of a DNA loop in vivo. Our findings illuminate how MukBEF can entrap DNA loops, and how these loops are primed for unloading from the complex.

99

Initial attempts to determine the structure of $E$. coli MukBEF were unsuccessful. We then the complex from Photorhabdus thracensis as a suitable candidate for structure determination by cryo-EM. The complex has $78 \%$ sequence identity to its $E$. coli homologue and stably co-purified with the E. coli acyl-carrier protein AcpP, an essential protein that is a binding partner of E. coli MukBEF (Niki et al., 1992; Prince et al., 2021). E. coli AcpP is $85 \%$ identical to $P$. thracensis AcpP. The purified complex had an estimated stoichiometry of $\mathrm{MukB}_{2} \mathrm{E}_{4} \mathrm{~F}_{2}-\mathrm{AcpP}_{2}$, which is a MukB $\mathrm{B}_{2}-\mathrm{AcpP}_{2}$ unit short of the $\mathrm{MukB}_{4} \mathrm{E}_{4} \mathrm{~F}_{2}$ dimer expected from

111 live-cell single-molecule microscopy (Badrinarayanan et al., 2012). We reasoned that this was either due to dissociation of MukBEF dimers during purification, or due to overproduction of 
MukEF leading to saturation and incomplete assembly of the complex. We therefore titrated

114 the preparation with $\mathrm{MukB}_{2}-\mathrm{AcpP}_{2}$ to reconstitute intact MukB $\mathrm{B}_{4} \mathrm{~F}_{2}$ dimers. This almost quantitatively shifted MukBEF to a smaller elution volume in size exclusion chromatography (SEC), indicating the formation of the physiological complex (Figure 1A).

To gain insights into how MukBEF interacts with matS sites during chromosomal unloading, we purified $P$. thracensis MatP, identified a cognate high-affinity matS site (Figure S1) and introduced the E1407Q mutation (hereafter MukB ${ }^{\mathrm{EQ}}$ ) into MukB to slow down ATP hydrolysis (Woo et al., 2009). We then reconstituted a complex between MukB ${ }^{\mathrm{EQ}} \mathrm{EF}$ dimers, MatP, and an 80 bp DNA oligonucleotide containing matS close to one end, in the presence of ATP and magnesium ions (Figures $\mathbf{1 B}$ and $\mathbf{1 C}$ ). The sample was then imaged by cryo-EM in vitreous ice

(Figure 1D).

We obtained a reconstruction of the DNA-bound MukBEF monomer part at an overall nominal resolution of $4.6 \AA$ (Figures $\mathbf{1 E}$ and S2). Focussed classification and refinement produced a map of $3.1 \AA$ resolution for the more rigid head module, with clearly resolved ATP and magnesium ions mediating head engagement (Figure 1F, S3A). This allowed the construction

130 of a complete atomic model for the complex, facilitated by previous crystallographic 131 information for individual parts (PDB: 3EUH, 3EUJ, 3IBP, 3VEA, 6H2X) (Bürmann et al., 2019;

132 Dupaigne et al., 2012; Kreamer et al., 2018; Li et al., 2010; Woo et al., 2009).

134 MukBEF adopts a compact and highly asymmetric conformation, with its ATPase heads bridged by the kleisin MukF (Figure 1E). The heads and hinge of MukB are brought into 
proximity by folding at the elbow. In addition to the elbow and the 'joint' at the heads

137 (Diebold-Durand et al., 2017), the MukB arms contain several other coiled-coil discontinuities

138 (Weitzel et al., 2011). Plasticity in these regions allows $\kappa$-MukB and v-MukB to adopt radically different conformations and thus break homodimer symmetry (Figure 1G).

141 The head-proximal arms of MukB are opened to allow accommodation of two distinct DNA

142 double helices (Figures $\mathbf{1 E}-\mathbf{1 F}, \mathbf{1 H}$ and $\mathbf{2 A}$ ). One DNA is bound by MatP and threads through

143 the inter-arm space near the joint. The other is clamped by the MukB heads, MukF, and MukE.

144 The chain of the kleisin MukF is resolved between residues 22-440, which represents $95 \%$ of

145 the protein (Figures $\mathbf{1 F}$ and $\mathbf{2 B}$ ). It partitions the two DNAs into topologically separated 146 compartments, the 'ring' delimited by MukF and the MukB arms, and the 'clamp' delimited

147 by MukF and the MukB heads (Figures $\mathbf{1 E}$ and $\mathbf{1 H}$ ). The DNA double helices have a crossing 148 angle of $60^{\circ}$, which is close to what has been estimated for negatively supercoiled 149 plectonemes (Rawdon et al., 2016). This suggests that in the context of an intact chromosome 150 they may originate from a single plectonemic loop, with MukBEF binding across the long axis of the loop (Figure 1H). This hypothesis will be explored below.

\section{The clamp contacts DNA across all core subunits}

The clamp has a highly asymmetric architecture imposed by the kleisin MukF. MukF is comprised of a C-terminal winged-helix domain (cWHD), a four-helix bundle forming the middle domain (MD), and an N-terminal winged-helix domain (nWHD). The cWHD and MD are connected by a 64 amino acid linker, which contains the MukE binding sites (Figure 2B). 
interface in other SMC complexes (Bürmann et al., 2013; Haering et al., 2004; Hassler et al., 2019; Woo et al., 2009). The MD which can bind the MukB neck (Zawadzka et al., 2018) is in a position roughly equivalent to binding sites between the kleisins and v-SMCs of cohesin, condensin and Smc-ScpAB (Bürmann et al., 2013; Gligoris et al., 2014; Hassler et al., 2019)

(Figure S3B). The MD is, however, structurally unrelated to the $\mathrm{N}$-terminal alpha-helical domain (nHD) of these kleisins.

MukB forms a homodimer, thus both MukB subunits contain MukF binding sites at cap and neck. However, the $v$-MukB does not form the cap/cWHD interface and $\kappa$-MukB does not associate with an MD. This asymmetric configuration is enabled by two separate steric occlusion mechanisms. At the v-MukB cap, binding of the MukF linker to $v$-MukB prevents binding of a cWHD, as has been observed before (Woo et al., 2009) (Figures $\mathbf{1 F}$ and 2B). In addition, the neck of $\kappa-M u k B$ is occluded by the hinge (Figure 1E), which prevents binding of a MD. These mechanisms preclude recruitment of additional MukEF subunits to the complex and thus prevent chaining of MUkBEF monomers into higher order polymers.

Within the clamp, all core subunits of MukBEF are in contact with DNA. The MukF linker is guided over the clamped DNA by the MukE dimer, which also binds DNA along its central cleft

177 (Figures 2A and 2B). The linker itself contacts the phosphate backbone with R322 and R327

178 (Figure 2C). The MukB heads bind the clamped DNA along their top surface (Figure 2D). The 179 'larynx' of v-MukB provides additional DNA contacts with Q1327 and R1328 (Figure 2B). This 
proteins (Figure S3C). Interestingly, the nHD of cohesin's kleisin Rad21 provides DNA contacts

that are located in a position similar to the larynx (Figure S3B).

The overall architecture of the MukBEF clamp appears analogous to what has been observed for the nuclease clamp in SbcCD (Rad50-Mre11) and the HAWK clamp in cohesin (Collier et

MukE is unrelated to any subunit in these complexes, however, other KITE-based SMC

We conclude that DNA binding on top of the ATPase heads is a common principle between

topologically equivalent clamps.

Whereas the clamped DNA is contacted by all core subunits of the complex, the DNA inside the ring is mostly bound by MatP, with only K1178 in the MukB joint contacting the phosphate backbone. The MatP dimer recognizes matS by inserting its $\alpha 4$ and $\beta 1$ elements into the major grove, as has been determined for MatP-matS complexes in isolation (Dupaigne et al., 2012) (Figure S1C). The C-terminal tetramerization tail of MatP, however, is not visible and 
The bridge is formed by residues between $\mathrm{H} 38$ and D42 in MatP and an N-terminal tail of MukE (Figure 2E). The latter involves residues between S2 and Q8, which are disordered in the second MukE subunit and also in previous crystal structures (Gloyd et al., 2011; Woo et al., 2009). The bridge interface is small and likely prone to dissociation, consistent with the finding that recombinantly overexpressed MukEF does not co-immunoprecipitate with purified MatP (Nolivos et al., 2016). This suggests that the bridge may have a transient role during unloading and dissociates once the reaction is complete, permitting the release of MukBEF from matS sites.

214 The joint of MukB is located at a central region of the complex. It is formed by an 84 amino acid insertion into the C-terminal coiled-coil strand and forms a slightly larger domain than the joints found in other SMC proteins (Figure S3C). The joint binds and positions MatP between the MukB arms (Figures $\mathbf{2 A}$ and $\mathbf{2 F}$ ). This interface is much larger than the MukEMatP bridge, and likely provides the major binding energy for association with MatP. The joint also provides a docking site for the hinge-proximal arm, with $L 605$ of $v$-MukB inserted between E1137 and Y1140 of א-MukB (Figure 2G). This likely contributes to stabilization of the elbow-folded conformation of MukBEF. and Y1103 and R1122 on the C-terminal helix (Figure 2G). Weak density protrudes from S36 of AcpP, which we have modelled as phosphopantetheine (PNS), the prosthetic group of AcpP 
227 (Cronan, 2014). At the $\kappa-M u k B$ binding site, PNS projects towards the space between the

228 head-proximal arm of $\kappa-M u k B$ and the hinge-proximal arm of $v$-MukB. The phosphate group

229 of PNS is in contact with R839 of $v$-MukB. PNS is modified with acyl moieties during fatty acid

230 synthesis, and although the functional role of AcpP within the MukBEF complex is unclear, it

231 may have a regulatory role coupling metabolism to chromosome organization (Gully et al.,

232 2003). Due to its position near the joint-arm contact, it is possible that AcpP, perhaps via its modification state, could have an influence on the elbow-folded state of MukBEF.

The joint is situated near the heads and is thus expected to be a central conduit for

conformational changes imposed by the ATPase cycle. Consistent with this idea, AcpP binding at the joint strongly increases MukBEF ATPase activity (Prince et al., 2021). Release of MukBEF from matS will likely require detachment from MatP, hence MatP binding at the joint seems ideal for regulation by the ATPase cycle, as will be explored below.

242 In the same sample that produced reconstructions of MukBEF bound to MatP-matS we also

243 observed particles with disengaged heads and neither DNA nor MatP bound (Figure 3A).

244 Although the map was resolved to only $6.8 \AA$, which prevented determination of the nucleotide state, it was very similar to exploratory reconstructions of nucleotide-free MukBEF

246 (Figure S2A). Hence, we refer to it as the 'apo state'. The apo complex is comparable in size

247 and shape to apo yeast condensin, with arms fully juxtaposed (Figure S3E). The apo clamp is

248 more flexible because it is not held in place by ATP and DNA, but clear density was observed at lower contour levels that allowed unambiguous positioning of MukEF. The map also 
revealed density for the second monomer within the context of a MukBEF dimer. Further classification produced a low-resolution map for the apo dimer, from which we obtained a model by rigid body fitting (Figure 3B).

The MukBEF dimer is held together by an extensive interface between MukF's MD and nWHD, which was observed previously by crystallography (Fennell-Fezzie et al., 2005; Woo et al., 2009). The two MukBEF monomers associate head-to-head with their MukEF subunits on the same face of the dimer. The complex thus has a front and back along its two-fold symmetry axis (Figure 3B).

259 In addition to dimers in the apo state, dimers associated with MatP and DNA were also readily resolved (Figure $3 \mathrm{C}$ ). Because we positioned the matS site close to one end of the $80 \mathrm{bp}$ DNA used for sample preparation, two dimers were able to associate with four DNAs in a 'tetrad' arrangement. Different classes of tetrads allowed us to assess the distance between the two dimers. We observed dimers distantly bridged by the DNA molecules (Figure $3 \mathrm{C}$ ) and closely apposed (Figure 3D). This is explained by sequence independent binding of the clamp, which can associate with any position along the DNA double strand (Figure 3E). Assuming that the clamp binds DNA not only during unloading at matS but also during a tentative translocation reaction, it may thus step or slide along the DNA track. Since the DNAs are roughly aligned with the dimer symmetry axis, it is conceivable that DNA translocation may operate along this axis. 
273 The MukB ${ }^{\mathrm{EQ}} \mathrm{EF}-\mathrm{MatP}-$ matS complex is blocked in ATP hydrolysis and shows a state prior to

274 unloading. The apo form, however, lacks both MatP and DNA and therefore represents the

275 result of a completed unloading reaction. Comparison of the two states should yield insights

276 into conformational changes that take place during MatP-dependent exit of DNA.

278 Sub-classification of the cryo-EM dataset revealed additional forms of MukBEF-MatP with 279 arms in different states of openness (Figure 4A). This suggests that the arms can gradually 'zip up', similar to what has been proposed for Smc-ScpAB based on distance measurements by electron paramagnetic resonance (Nunez et al., 2021). The most open class has arms unzipped up to the elbow, and the most closed one is the apo state. In the apo state, the heads disengage and tilt, and the joints and larynx become closely juxtaposed (Figure 4B). This occludes the binding sites for both MatP-matS and the clamped DNA and strongly supports the idea that ATP hydrolysis promotes dissociation from MatP-matS and DNA unloading.

Conformational changes associated with the release of nucleotide and DNA propagate through the whole complex and can be observed even in the hinge-proximal coiled coil

(Figure 4C). The hinge follows the tilting $\kappa-M u k B$ neck during DNA unloading, and the hingeproximal arm changes from a straightened conformation to a strongly curved one. This indicates that the arm stores parts of the binding energy provided by ATP, MatP and DNA as elastic energy, similar to a spring. This energy may be harnessed to expel MatP and DNA after ATP hydrolysis. 
In cohesin, DNA unloading proceeds via opening of the interface between the kleisin Scc1 and

MukBEF, the corresponding interface, which we name 'neck gate', is formed by the neck of

Intriguingly, the neck gate is opened by a narrow cleft along the interface in the MatP/DNA opening of the neck gate.

308 MukBEF adopts an elbow-folded conformation, at least in its apo state and when bound to

309 ATP, MatP and DNA. However, the elbow has also been crystallized in an extended 310 conformation, suggesting that MukBEF may convert to extended rods or fully open rings with

311 disengaged arms (Bürmann et al., 2019) (Figure 5A). The hinge-proximal arms are in a closed

312 rod conformation in our structures, similar to those of other SMC complexes (Figure S3F), but

313 a conformation compatible with open rings has been observed by crystallography (Li et al.,

314 2010) (Figure S3G). Additional support for the existence of open rings comes from rotary shadowing electron microscopy experiments (Matoba et al., 2005). We thus decided to clarify

316 whether the elbow-folded conformation is abundant in vivo, and whether it may be 
controlled by the ATPase cycle of MukBEF. To accomplish this, we probed the conformation of endogenous E. coli MukBEF by site-specific cysteine cross-linking.

To introduce multiple point mutations spread across the $8 \mathrm{~kb}$ chromosomal mukFEB locus, we used a derivative of REXER (replicon excision for enhanced genome engineering through programmed recombination) (Wang et al., 2016). In addition to introducing mutations A304C and D857C into MukB to probe the folded conformation (Figure 5A) we introduced C1118S, which ablated weak background cross-linking with a small protein, possibly AcpP (Figure S4). Next, we treated the E. coli cells with bismaleimidoethane (BMOE), which rapidly in vivo crosslinks closely spaced thiols such as cysteine sidechains. We then detected reaction products labelled with HaloTag-tetramethylrhodamine (TMR) by SDS-PAGE and in-gel fluorescence

(Figure 5B). Residues A304C and D857C cross-linked specifically with close to $50 \%$ efficiency, demonstrating that the folded conformation exists in vivo. The reaction efficiency was comparable to that of constitutive interfaces (see below), indicating that the folded state is abundant.

Next, we generated ATPase mutant strains S1366R (MukB ${ }^{\text {SR }}$, blocking head engagement), D1406A (MukB DA, blocking ATP binding) or E1407Q (MukB ${ }^{\mathrm{EQ}}$, blocking ATP hydrolysis) (Woo et al., 2009) (Figure S5A). As expected, all mutations conferred a mukB null phenotype, characterized by an inability to grow on rich media at $37{ }^{\circ} \mathrm{C}$. To probe the effect of the mutations on the ATPase cycle, we then introduced G67C, which is located at the top of the MukB head and close to its symmetry mate in the second MukB (Figure S5A). This residue 
cycle. The residue cross-linked with similar efficiencies in WT and the ATP binding mutant

MukB $^{\mathrm{DA}}$, with $22 \pm 1 \%$ and $21 \pm 2 \%$, respectively. The cross-linked fraction was increased to of wild-type MukBEF are mostly disengaged.

We then probed for elbow folding in $\mathrm{MukB}^{\mathrm{SR}}$, MukB ${ }^{\mathrm{DA}}$ and $\mathrm{MukB}^{\mathrm{EQ}}$ strains which resulted in structural data. context of a circular chromosome, DNA would have to enter through one or more entry gates. For biochemical preparation of the cryo-EM sample, however, any loading or partial unloading reactions were bypassed using linear DNA and an ATPase-deficient mutant. As an additional caveat, linear DNA prevents determination of the DNA connectivity that would originate from the same or from different chromosomes. Hence, we decided to map the DNA binding topology of MukBEF in vivo. 
this assay, covalent circularization of a protein compartment around chromosomal DNA clamp (Figures 6B and S5A). In addition, head and hinge cysteines were combined to probe entrapment in the 'frame' compartment, which is the union of ring and clamp.

372 Combinations of cysteines were introduced into the endogenous mukFEB locus, cells were treated with BMOE, and reaction species were identified by SDS-PAGE and in-gel fluorescence

(Figure 6C). Cross-linking was specific and efficient at all sites. For some reaction products, mass and mere shape differences. However, depletion of precursors in expected ratios indicated successful multi-site reactions in all cases. For example, the MukB species crosslinked at the heads was reduced from $29 \%$ to $12 \%$ when combined with the hinge cross-link. This corresponds to a reduction by $60 \%$ and is in excellent agreement with the $62 \%$ crosslinking efficiency observed for the hinge.

382 First, we tested entrapment in the ring compartment using cap, neck, and hinge cysteine pairs. We treated cells with BMOE, lysed them in agarose plugs to protect chromosomal DNA

384 from shearing and subjected plugs to electrophoresis in the presence of $0.1 \%$ sodium dodecyl sulphate (SDS). This denatures and extracts proteins and only retains cross-linked species that 
have been circularized around DNA. We then digested chromosomal DNA and isolated the eluted proteins. A cross-linked high-molecular weight MukB species was retained only when cap, neck and hinge interfaces all contained cysteine pairs, indicating that it is the covalently circularized $\mathrm{MukB}_{2} \mathrm{~F}$ (Figure 6D). The species was not retained from MukB ${ }^{\mathrm{DA}}$ or MukB ${ }^{\mathrm{EQ}}$ strains, which suggests that DNA entrapment critically depends on ATP hydrolysis.

Next, we tested for DNA entrapment in the clamp compartment using cap, neck, and head was isolated as the major band, accompanied by small amounts of protein presumably resulting from chemical cross-link reversal during the protein isolation procedure (Shen et al., 2012; Wilhelm and Gruber, 2017). The retained amount was similar to that of the ring compartment species, consistent with the notion that both clamp and ring entrap DNA simultaneously. Protein was not retained from MukB ${ }^{\mathrm{DA}}$ or MukB $\mathrm{MQ}^{\mathrm{EQ}}$ strains. This finding indicates that DNA entrapment in the clamp, as in the ring, depends on ATP hydrolysis in vivo.

Next, we tested for DNA entrapment in the frame compartment using the combination of

405 Cross-linking the frame around this loop allows DNA to slip out of the covalent protein circle, 
408 Taken together, these results fully support entrapment of a loop as suggested by the structure

409 (Figures 1H, 6G and S6B-S6C). Because no DNA entrapment is detected in the frame 410 compartment, and signals for clamp and ring are similar, most if not all complexes with DNA

411 in the clamp must have DNA catenated with the ring, and vice versa. The results are

412 incompatible with entrapment in only ring or clamp, with entrapment of sister chromosomes,

413 or with a loop axis running parallel to the plane of the ring (Figure S6C). Any of these forms

414 would lead to catenation with the frame compartment.

415

416 Finally, we investigated whether chromosome entrapment was dependent on MatP. Deletion

417 of the matP gene had little if any effect on DNA inside the MukBEF ring (Figure $6 \mathrm{H}$ ). We

418 suggest that DNA entrapment is a more general feature of MukBEF and does not exclusively

419 occur during unloading at matS sites.

420

421

Discussion

422

The double-locked loop

423 The structure of MukBEF bound to MatP-matS revealed the simultaneous entrapment of two

424 DNA double helices, topologically separated into ring and clamp compartments. We name

425 this configuration the 'double lock' (Figure 7A). The DNA crossing angle in the MatP-bound

426 double lock indicates that a DNA loop passes through MukBEF with the loop long axis

427 perpendicular to the ring plane. Topological mapping in vivo supports this notion. 
DNA entrapment by MukBEF occurs mainly outside of the MatP-matS context. Because happens throughout the chromosome. At matS sites, MatP may enhance the positioning of

How abundant is the double lock? The fraction of cross-linked species retained by the

440 likely underestimates abundance by an unknown and possibly large factor, and may thus not

be suited for its quantification. In other words, the data support the existence of the double lock. 
MukBEF monomers was an asymmetric process, similar to what has been observed for monomeric condensin (Ganji et al., 2018), such side-by-side coupling could symmetrize the overall process. Highly asymmetric loop extrusion is considered a hindrance for physiological chromosome folding and may be resolved using a dimerization mechanism (Banigan and Mirny, 2019). We suggest that the arrangement of the MukBEF dimer is well suited to implement symmetric loop extrusion.

MukBEF does not entrap sister DNAs or loops with a long axis perpendicular to the one proposed (Figure S6C). However, the low sensitivity of our assay may have precluded topological' loops or 'non-topological' loops, which may form in addition to the double lock. constituting a protein-DNA rotaxane instead of a catenane, whereas the latter do not thread through the complex at all but bind to its outer surface (Figure S6C). These structures may be involved in loop extrusion by cohesin (Davidson et al., 2019; Srinivasan et al., 2018), but their binding by MukBEF is hypothetical. If 'pseudo-topological' loops exist, however, they will need to be accommodated in the ring and not in the clamp due to space limitations (Figure

470 S6B). Whereas the clamp is highly constrained by a short and compact MukEF and can thus

471 accommodate only a single DNA, the ring would be able to embrace multiple DNAs and could 472 even enlarge its capacity by extending the elbow and fully opening the arms. 
474 Cysteine cross-linking has been used to map DNA entrapment in other SMC complexes

475 (Chapard et al., 2019; Gligoris et al., 2014; Haering et al., 2008; Vazquez Nunez et al., 2019;

476 Wilhelm et al., 2015). Cross-linking at cap, neck and hinge of cohesin and Smc-ScpAB (also

477 designated as 'SK' cross-links) retains either complex on DNA after denaturation. When cross-

478 linked at cap, neck and juxtaposed heads (also designated as 'JK' cross-links), DNA association

479 is also maintained. Entrapment is not observed when cysteines in hinge and juxtaposed heads

480 are combined (also designated as 'JS' cross-links). These patterns are topologically equivalent

481 to the ones determined here. Hence, the MukBEF structure may provide an attractive

482 interpretation for these observations, namely that cohesin and Smc-ScpAB may be able to entrap DNA in a manner similar to the double-lock configuration.

Interestingly, the head cross-links used for cohesin and Smc-ScpAB can capture a "head experiments, and the G67C cross-link at the heads used for topology mapping can capture the disengaged state. It is therefore possible that DNA may be retained in the clamp even after ATP hydrolysis. 
496

497

498

499

500

501

502

503

504

505

506

507

508

509

510

511

512

513

514

515

516

517 The proposed unloading model, which is purely based on structural data, is attractive for 518

represents the state before DNA has entered the complex. Models for chromosomal turnover of MukBEF will have to fit these observations.

We envision that unloading of matS from the ring is coupled to unloading of DNA from the clamp (Figure 7B). For the clamped DNA, the interface between the heads is a prime candidate for an exit gate because its formation is regulated by ATP binding and hydrolysis. Upon ATP hydrolysis and phosphate/nucleotide release, heads disengage and dissociate their DNA binding surfaces. Clamped DNA may then be able to exit via the cleft between disengaging heads. For this to happen, the MukF linker, which also seals the head gate, needs to detach from the $v$-MukB head. MukEF can stay bound to and move along with the DNA because the neck gate is open, which allows MukEF to reposition in relation to the $v$-MukB head. Concurrently, a deformation at the joints releases MatP, which stays associated with MukEF via the MatP-MukE bridge. Next, the head proximal arms of MukB zip up and occlude the binding sites for MatP and DNA at the joints and heads, respectively. Release of energy stored as deformations in the hinge-proximal arm will reinforce this process. MatP and DNA are ejected and are free to dissociate from the complex. Finally, closure of the neck gate reverts MukBEF to its apo form. We note that the proposed mechanism would not strictly depend on MatP-matS but could also eject 'free' DNA from the ring compartment. Its driving force comes from relaxation of MukB into the apo conformation, whereas MatP is a structural element that ensures ideal positioning of DNA close to the exit gate. several reasons. First, it explains how the process is regulated by ATP hydrolysis, namely by 
520 how the process is enhanced at matS sites. Third, the model designates the neck gate as the

521 topological exit gate of the tripartite ring. The equivalent interface in the distantly related

522 cohesin is the exit gate of this complex (Chan et al., 2012; Muir et al., 2020; Murayama and

523 Uhlmann, 2015). We suggest that unloading via the neck gate is a widely conserved activity

524 of SMC complexes.

525

526 An interaction of MatP with the MukB hinge, at least in the absence of DNA, has been

527 reported (Fisher et al., 2021; Nolivos et al., 2016) but is not seen in our structures. It is conceivable that this occurs at a different stage during unloading and could suggest subunit and DNA transport within the complex. Interestingly, MukB ${ }^{\mathrm{EQ}}$ can associate with matS in cells

530 (Nolivos et al., 2016), but DNA entrapment is not detected in our assay. This may point towards a state which binds MatP but does not entrap DNA.

In the light of our structures, DNA entry into MukBEF appears enigmatic, as is the case for

534 other SMC complexes. The joint, which has emerged as a central region regulated by the

535 ATPase, is required for recruitment of Smc-ScpAB to its loading factor ParB (Gruber and

536 Errington, 2009; Minnen et al., 2016). ParB is typically not present bacteria that use MukBEF,

537 and no substitute loading factor has been identified. Importantly, loading of MukBEF does not

538 depend on MatP, and a reverse and MatP-independent version of the unloading mechanism proposed above would have to work against a large entropic barrier. This rather unlikely 
541 hydrolysis. We suspect that chromosomal loading and unloading of MukBEF are achieved by

542 considerably different means.

543

544 Outlook

545 Here we have determined the structure of MukBEF both in its apo state and in a

546 MatP/DNA/ATP bound form, providing molecular insight into how MukBEF is released from

547 chromosomes. This led to the finding that MukBEF can entrap DNA loops in a double-lock

548 configuration, which links two important topological concepts, namely DNA entrapment

549 inside the ring and inside the clamp compartment. Our work opens up the questions of how

550 the double-locked loop is established, and how MukBEF operates on this and possibly other

551 types of DNA structures. We anticipate that biochemical reconstitution of the process by

552 which MukBEF organizes chromosomes, coupled to structural analysis, will further our

553 understanding of chromosome folding in bacteria and beyond.

554

555

Data availability

556 EM density maps have been deposited in the EMDB (EMD-12656, EMD-12657, EMD-12658,

557

EMD-12659, EMD-12660, EMD-12662, EMD-12663, EMD-12664). Coordinates have been

558 deposited in the PDB (7NYW, 7NYX, 7NYY, 7NYZ, 7NZO, 7NZ2, 7NZ3, 7NZ4). See also Table S1.

559 All other data will be available upon request. 


\section{Material \& Methods}

562

\section{Protein production and purification}

563

Wild-type P. thracensis MukBEF (NCBI accession identifiers WP_046975681.1,

WP_046975682.1, and WP_046975683.1) was produced from a polycistronic expression

565

construct assembled into a pET28 based backbone by Golden Gate cloning (Engler et al., 2008)

566

(pFB403). The construct contained a His6-SMT3 tag fused to residue 1 of MukB which allowed

567

affinity purification and scar-less tag removal by hSENP2 protease (Butt et al., 2005). The

568

complex was produced in BL21-Gold(DE3) by autoinduction (Studier, 2005) in ZYP-5052 media

569

at $24{ }^{\circ} \mathrm{C}$. All purification steps were carried out at $4{ }^{\circ} \mathrm{C}$. $15 \mathrm{~g}$ of cells were resuspended in 90

570

$\mathrm{mL}$ of IMAC buffer (50 mM Tris, $300 \mathrm{mM} \mathrm{NaCl}, 40 \mathrm{mM}$ imidazole, $1 \mathrm{mM}$ TCEP, $\mathrm{pH} 7.4$ at room

571

temperature (RT)) supplemented with protease inhibitor cocktail (Roche) and Benzonase

572

(Merck) and lysed at $172 \mathrm{MPa}$ in a high-pressure homogenizer. The lysate was cleared by

573

centrifugation at $96,000 \times \mathrm{g}$ for $30 \mathrm{~min}$, passed through a $0.45 \mu \mathrm{m}$ filter, and incubated for 30

574

min with $25 \mathrm{~mL}$ Ni-NTA agarose (Qiagen) equilibrated in IMAC buffer. The resin was packed

575

into a gravity flow column and washed with $3 \times 50 \mathrm{~mL}$ IMAC buffer, then resuspended in 25

576

$\mathrm{mL}$ IMAC buffer containing $1 \mathrm{mg}$ GST-hSENP2 and incubated for $1 \mathrm{~h}$ on a roller. Eluate was

577

collected and pooled with a $12.5 \mathrm{~mL}$ wash using IMAC buffer, diluted with $18.8 \mathrm{~mL}$ buffer $\mathrm{Q}$

578 (10 mM Tris, pH 7.4 at RT), passed through a $0.22 \mu \mathrm{m}$ filter and applied to a $20 \mathrm{~mL}$ HiTrap

579 Heparin HP column (GE Healthcare). MukBEF was largely found in the flowthrough and was

580 applied to a $5 \mathrm{~mL}$ HiTrap Q HP column (GE Healthcare). The column was washed with 2 column

581 volumes (CV) of $10 \mathrm{mM}$ Tris, $200 \mathrm{mM} \mathrm{NaCl}, 1 \mathrm{mM}$ TCEP, $\mathrm{pH} 7.4$ at RT, and protein was eluted

582 with a $20 \mathrm{CV}$ linear gradient from $200 \mathrm{mM} \mathrm{NaCl}$ to $1 \mathrm{M} \mathrm{NaCl}$ in buffer Q. MukBEF eluted at 
and was injected into a Superose 6 Increase 10/300 column (GE Healthcare) in buffer H200 (20 mM Hepes, $200 \mathrm{mM} \mathrm{NaCl}, 1 \mathrm{mM}$ TCEP, pH 7.3 at RT). Peak fractions were pooled, concentrated to $8.2 \mathrm{mg} / \mathrm{mL}$ on a Vivaspin $2 \mathrm{MWCO} 30$ filter, aliquoted, frozen in liquid nitrogen and stored at $-80^{\circ} \mathrm{C}$ until use. Protein stoichiometry was estimated by SDS-PAGE and Coomassie staining as $\mathrm{MukB}_{2} \mathrm{E}_{4} \mathrm{~F}_{2}-\mathrm{AcpP}_{2}$.

589

590 We were unable to establish polycistronic MukB ${ }^{\mathrm{EQ}} \mathrm{EF}$ expression constructs, likely due to toxicity in the cloning host. Therefore, we cloned $\mathrm{His}_{6}-\mathrm{SMT3}-\mathrm{MukB}{ }^{\mathrm{EQ}}$ and MukEF on two separate expression constructs, pFB485 and pFB486, whereby the His6-SMT3-MukB ${ }^{\mathrm{EQ}}$ construct was always propagated at $22^{\circ} \mathrm{C}$. Proteins were separately produced as above, with His $6-S M T 3-M u k B^{E Q}$ production at $22^{\circ} \mathrm{C}$. Cell pellets of both strains ( $15 \mathrm{~g}$ each) were mixed in $180 \mathrm{~mL}$ IMAC buffer, and the complex was purified as described above, except that $1 \mathrm{mM}$ EDTA ( $p H 7.4$ at RT) was added before application to the Heparin column with the intention to improve dissociation of potentially co-purifying nucleotides. Estimated stoichiometry was $\operatorname{MukB}^{\mathrm{EQ}} \mathrm{E}_{4} \mathrm{~F}_{2}-\mathrm{AcpP}_{2}$.

599

MukB $^{\mathrm{EQ}}$ was purified as above except for omission of the Heparin step. Protein was concentrated to $8 \mathrm{mg} / \mathrm{mL}$. Estimated stoichiometry was $\mathrm{MukB}^{\mathrm{EO}}{ }_{2}-\mathrm{AcpP}_{2}$.

602

P. thracensis MatP (NCBI accession identifier WP_046976581.1) was cloned without tag was prepared as above and was passed through a $5 \mathrm{~mL}$ HisTrap HP column (GE Healthcare). 
buffer containing $270 \mathrm{mM}$ imidazole. The eluate was diluted with an equal volume of buffer

615 All protein concentrations were determined by absorbance at $280 \mathrm{~nm}$ using theoretical absorption coefficients. Annotated sequences of expression constructs are provided in Data

617 S1.

620 Initial binding experiments indicated that the affinity of $P$. thracensis MatP for consensus $E$. coli matS GTGACATTGTCAC (palindrome underlined) was about an order of magnitude lower

622 than reported for other MatP proteins (Dupaigne et al., 2012; Mercier et al., 2008). To identify alternative matS sites, we mapped all sites with edit distance up to 2 within the palindromic 624 region onto the $P$. thracensis chromosome (NCBI accession identifier CP011104.1) using

625 Wolfram Mathematica, and ranked them by median distance to the replication terminus 626 predicted by cumulative GC skew (Lobry, 1996). This identified GTTACNNNGTAAC as an 627 abundant site with 9 occurences (Figure S1A) which was further characterized by EMSA. 
6-Carboxyfluorescein (6-FAM) labelled DNA oligonucleotides matS1 (annealed from single

630 stranded

oligonucleotides

[6-FAM]CACTGTGACATTGTCACGGCA

and

631

TGCCGTGACAATGTCACAGTG; E. coli consensus mats underlined) or matS2 ([6-

FAM]CACTGTTACAGTGTAACGGCA and TGCCGTTACACTGTAACAGTG; $P$. thracensis candidate

633

site underlined) at a final concentration of $2 \mathrm{nM}$ in buffer $\mathrm{H} 30$ (20 mM Hepes, $30 \mathrm{mM} \mathrm{NaCl}, 1$

634

mM TCEP, pH 7.3 at RT) were titrated with MatP and incubated for 5 min at RT. Samples were

635

resolved on a $6 \%$ DNA Retardation gel (Thermo Fisher Scientific) with $0.5 x$ TBE running buffer

636

at $100 \mathrm{~V}$ for $60 \mathrm{~min}$ at $4{ }^{\circ} \mathrm{C}$. Gels were scanned on a Typhoon TLA9000 with Cy2 setup.

637

Quantification of bands was performed with Wolfram Mathematica using moving median

estimation of background signal. Data were fit by a rate equation model at equilibrium in an

640

arbitrary time domain, parametrized by dissociation rate $k_{d}$, baseline and asymptote, and with

641

the association rate $k_{a}$ as an arbitrary model constant. The equilibrium dissociation constant

642

$K_{d}$ was determined as $k_{d} / k_{a}$ which is independent of the time domain. This approach is easily

643

extendable to reaction models where analytical solutions or approximations are difficult to

644

derive and can be adapted to time resolved detection methods for determination of true rate

645 constants.

648 Optimal protein ratio for formation of MukBEF dimers was estimated by titrating $375 \mathrm{nM}$

$649 \mathrm{MukB}_{2} \mathrm{E}_{4} \mathrm{~F}_{2}-\mathrm{AcpP}_{2}$ with $\mathrm{MukB}_{2}-\mathrm{AcpP}_{2}$ in buffer $\mathrm{H} 200(20 \mathrm{mM}$ Hepes, $200 \mathrm{mM} \mathrm{NaCl}, 1 \mathrm{mM}$

650 TCEP, pH 7.3 at RT), followed by size exclusion chromatography on a Superose 6 Increase

$6513.2 / 300$ column (GE Healthcare) mounted on an ÄKTA Ettan (GE Healthcare). An equimolar 
mixture shifted most protein to the dimer fraction, with some residual material in $\mathrm{MukB}_{2} \mathrm{E}_{4} \mathrm{~F}_{2}-$ $\mathrm{AcpP}_{2}$ and $\mathrm{MukB}_{2}-\mathrm{AcpP}_{2}$ fractions.

654 mM Hepes, $200 \mathrm{mM} \mathrm{NaCl}, 1 \mathrm{mM}$ TCEP, pH 7.3 at RT) and incubated for $10 \mathrm{~min}$ on ice. Then, CTCGCCTGTAAAGTAGGCATTAGTTGTTCGTAGTGCTCGTCTGGCTCTGGATTACCCGCCACTGTTAC H200) were added. After 5 min on ice, $4.9 \mu \mathrm{L}$ of buffer H30 (20 mM Hepes, $30 \mathrm{mM} \mathrm{NaCl}, 1$ mM TCEP, pH 7.3 at RT) were added and the sample was passed over a Zeba Micro Spin 7K MWCO column (Thermo Fisher Scientific) equilibrated in buffer H30 containing 1 mM ATP, 2 $\mathrm{mM} \mathrm{MgCl} 2$ and $0.05 \%(\mathrm{w} / \mathrm{v}) \beta$-octyl glucoside. The sample was incubated for $10 \mathrm{~min}$ at $\mathrm{RT}$, then for 30 min on ice.

667 mesh grids (Quantifoil) were glow discharged for $15 \mathrm{~s}$ at $30 \mathrm{~mA}$ in a Quorum SC7620, and 2.5 $\mu \mathrm{L}$ sample were applied to a freshly glow discharged grid mounted in a TFS Vitrobot Mark IV,

671 equilibrated at $4{ }^{\circ} \mathrm{C}$ and $100 \%$ humidity. Grids were immediately blotted at blot force $-15,2$ -

$6724 \mathrm{~s}$ blotting time, no drain time and plunge frozen in liquid ethane. Grids were stored in liquid 673 nitrogen until use. Sample screening and optimization was performed on TFS Tecnai F20, Polara and Glacios microscopes. 
677 Data was collected on multiple grids over three sessions on a TFS Titan Krios with X-FEG emitter at $300 \mathrm{kV}$, equipped with a Gatan K3 detector operating in counting mode and a Gatan

679 Quantum energy filter with $20 \mathrm{eV}$ slit width centered on the zero-loss peak. Datasets 1 and 2 were collected with hardware binning at $1.07 \AA$ A calibrated pixel size. Dataset 3 was acquired

681 without hardware binning at $0.535 \AA$ calibrated pixel size. Movies were acquired in SerialEM 682 (Mastronarde, 2005) at three areas per hole, using image shift with beam tilt compensation 683 to collect 9 holes per stage movement. Target defocus was -1 to $-3 \mu \mathrm{m}$, total electron fluence was $40 \mathrm{e}^{-} / \mathrm{A}^{2}$ collected over $2.5 \mathrm{~s}$ and fractionated into 40 frames.

685

687 An overview of the data analysis workflow is shown in Figure S2. Motion correction and dose weighting was performed in RELION (Scheres, 2012) with one patch per micrograph and onthe-fly gain correction. Super-resolution data of dataset 3 were binned by a factor of 2 . The contrast transfer function (CTF) was fitted with CTFFIND4 (Rohou and Grigorieff, 2015). Particle picking was performed with crYOLO (Wagner et al., 2019). All further processing was done in RELION.

An initial model of MukBEF was reconstructed from an exploratory dataset collected on apo$\mathrm{MukB}_{2} \mathrm{E}_{4} \mathrm{~F}_{2}-\mathrm{AcpP}_{2}$ (Figure S2A). Particles were picked with a custom trained crYOLO model and subjected to 2D classification. Particles from good classes were used for $a b$ initio reconstruction. The model was manually sculpted at to remove obvious artefacts, and 
iteratively improved by 3D classification, auto-refinement, sculpting, optimization of the crYOLO model and repicking.

700

701

MukB ${ }^{\mathrm{EQ}} \mathrm{EF}-\mathrm{MatP}-\mathrm{DNA}$ particles were picked from dataset 1 using the apo-MukBEF crYOLO

702

model. Using the apo-MukBEF reconstruction as a starting model, an initial model for

703

MukB ${ }^{\mathrm{EQ}} \mathrm{EF}-\mathrm{MatP}-\mathrm{DNA}$ was obtained after rounds of refinement, 2D classification without

704

alignment, 3D classification with global pose search and masked 3D classification without

705

alignment focused on the joint (Figure S2A). Next, particles from all datasets were extracted

706

at $4.3 \AA / p x$ and partitioned into optics groups by hole position and grid. The initial model was

707

separately refined against batches of particles, and batches were cleaned by 2D classification

708

without alignment. Particles were re-extracted with re-centering at $4.3 \AA / p x$, and the initial

709

model was refined against all of them. Particles were then subjected to 3D classification

710

without alignment. The best class showed residual density for additional monomers arranged

711

in a tetrad (Figures $3 \mathbf{C}$ and S2A). Monomers of the tetrad were extracted at $1.45 \AA / p x$ and

pooled. Refinement with global pose search, followed by duplicate removal and refinement

713

with local pose search resulted in a map at 6.2 Å resolution. This was further classified without

alignment using a mask around the arms, followed by signal subtraction of the head module,

715 and focused refinement and 3D classification without alignment of the elbow region. After

716 reverting to original particles, focused refinement of the head module followed by CTF

717 refinement (beam tilt, anisotropic magnification, per-particle defocus) and Bayesian polishing were performed. A final refinement that contained the full complex inside its mask yielded a map at an overall nominal resolution of $4.6 \AA$. 
Reconstructions of the open states and apo complex as shown in Figure 4A were obtained by dimer and DNA bound tetrads (Figure 3B-3D) were obtained by re-centering and subclassification of classes from the main tree.

A focused reconstruction of the MukBEF-MatP-DNA head module was obtained as follows. monomers within a tetrad were extracted with re-centering, subjected to multiple rounds of 3D classification, 3D refinement, CTF refinement (beam tilt, anisotropic magnification, perparticle defocus) and a final round of Bayesian polishing. Duplicate removal was performed on multiple occasions to exclude the same head in a tetrad contributing multiple times. At final stages, refinement was performed with local pose search. Using a mask around the head module, this resulted in two maps, one from pooled datasets 1 and 2 at $3.5 \AA$ A resolution, and one from dataset 3 at $3.4 \AA$ resolution. After pooling particles, the combined refinement yielded a map at $3.3 \AA$ resolution. Re-centering on the holocomplex with box expansion, followed by CTF refinement and Bayesian polishing yielded a final map at $3.1 \AA$ resolution. 
743 First, a model for the head module was built into its map at 3.1 Å resolution, sharpened by B-

744 factor compensation and FSC weighting (Rosenthal and Henderson, 2003) in RELION

745 PostProcess. Homology models were obtained from PDB entries 3EUJ, 3EUH, 3VEA and 3IBP

746 (Dupaigne et al., 2012; Li et al., 2010; Woo et al., 2009) using SWISS-MODEL (Waterhouse et

747 al., 2018), and rigid body fitted using ChimeraX. PDB entry 6DFL was used as a starting model

748 for AcpP (Kreamer et al., 2018). The hinge-proximal arm region was flexibly fitted in ISOLDE

749 (Croll, 2018). The model was then partially rebuilt in Coot (Emsley et al., 2010), whereby the

750 necks, joints and parts of the hinge-proximal arm were built de novo. The clamped DNA was

751 modelled as poly-AT. Next, the model was subjected to phenix.real_space_refine (Afonine et

752 al., 2018) to resolve major clashes, and annealed using ISOLDE. The model was improved by

753 cycles of editing in ISOLDE and Coot, and automated refinement in phenix.real_space_refine

754 using secondary structure restraints (alpha, beta, base-pair), Ramachandran restraints,

755 without non-crystallographic symmetry (NCS) constraints. Model vs. map FSC was computed

756 in PHENIX and is shown in Figure S2C.

757

758 A medium resolution model for the complete MukBEF-MatP-DNA monomer was built as

759 follows. The head module was rigid body fitted into the $4.6 \AA$ holocomplex map sharpened in

760 RELION PostProcess. Homology models for hinge and elbow were obtained from PDB entries

761 3IBP and 6H2X (Bürmann et al., 2019; Li et al., 2010), respectively, using SWISS-MODEL.

762 Homology models were split and flexibly fitted using ISOLDE. Connecting segments between

763 head-and hinge-proximal arms and the elbow coiled coil were built de novo. There was little

764 or no sidechain information for these segments, but their simple coiled-coil architecture and 
highly constrained ends allowed building of a realistic model. For example, residues of the predicted hydrophobic heptad-repeat patterns locate to the helix interfaces, and prolines locate to helix breaks. The model was improved by editing in ISOLDE and Coot, and automated refinement in phenix.real_space_refine using reference model restraints for hinge and head module, secondary structure restraints, Ramachandran restraints, and no NCS constraints. Model vs. map FSC was computed in PHENIX and is shown in Figure S2C.

773 fitting of the holocomplex reference model in ISOLDE followed by automated refinement in phenix.real_space_refine.

Low resolution models for dimers and tetrads were obtained by rigid body fitting of holocomplex monomers into multimer maps blurred to $20 \AA ̊$ resolution. Connecting peptides in MukF were built in Coot with a homology model of the MukF dimer based on PDB entry 3EUH (Woo et al., 2009) as a guide. The models contained some clashes at the MukF

780 interfaces, some of which were resolved by changing sidechain rotamers without touching

781 the main chain. Next, idealized 80 bp double-stranded DNA was generated in Coot and flexibly

782 fitted in ISOLDE using strong distance restraints. Protein-bound regions of the DNA were replaced by the corresponding parts of the rigid-body docked medium resolution DNA models. The head bound poly-AT models were edited to match the oligonucleotide sequence. DNA was subjected to a single cycle of phenix.real_space_refine with base pair and input 
787

788

789

790

791

792

793

794

795

796

797

798

799

800

801

802

803

804

805

806

807

apposed (Figure 3D), only two monomers were modelled due to weak density for the remaining two monomers.

Models were rendered in ChimeraX. Model statistics are listed in Table S1.

\section{E. coli strains}

Strains are based on E. coli MG1655 and are listed in Table S2. All strains were viable in LB at $37{ }^{\circ} \mathrm{C}$, except for $\triangle m u k B, \operatorname{mukB}(\mathrm{S} 1366 \mathrm{R}), \operatorname{mukB}(\mathrm{D} 1407 \mathrm{~A})$ and mukB(E1406Q) derivatives which were cultivated at $22^{\circ} \mathrm{C}$, their permissive temperature. The strain containing cap, neck, and head cysteines for circularization of the clamp compartment (SFB202) was viable at $37^{\circ} \mathrm{C}$, but grew with a reduced rate, whereas all other strains with functional alleles grew with rates similar to WT. Pre-cultures for all experiments were grown side-by-side to stationary phase and stored at $4{ }^{\circ} \mathrm{C}$ for up to two weeks.

\section{Genome engineering for strain construction}

For construction of acceptor strains we integrated a pheS(T251, A294G)-hyg ${ }^{R}$ double selection cassette either downstream of the mukFEB operon (SFB047, SFB180) or replacing the mukFEB coding region (SFB053) by $\lambda$-Red recombineering (Yu et al., 2000). pheS(T251A, A294G) is a robust negative selection marker that encodes a mutant phenylalanyl-tRNA synthetase which confers toxicity in presence of 4-chloro-phenylalanine (4-CP) (Miyazaki, 2015). The locus was then targeted using a REXER-based strategy to introduce a C-terminal HaloTag on mukB and desired point mutations (Wang et al., 2016 and in preparation). The modified locus was linked 
to a $n e o^{R}$ cassette downstream of the mukFEB transcriptional terminator. Recombinants were selected on media containing $12.5 \mu \mathrm{g} / \mathrm{mL}$ kanamycin and $2.5 \mathrm{mM}$ 4-CP.

811

812 A marker-free $\triangle$ matP allele was constructed by replacing matP with pheS(T251, A294G)-hyg ${ }^{R}$ and subsequent cassette ejection with a double-stranded oligonucleotide coding for a matP

814 in-frame deletion.

815

816 All strains were single colony purified, and verified by marker analysis, phenotype, PCR, and

817 Sanger sequencing where appropriate.

820 Cells were grown to exponential phase in LB (OD 0.2-0.3) if not indicated otherwise. Cultures 821 were mixed with $30 \%(w / v)$ ice, harvested by centrifugation, and kept cold for the duration 822 of the experiment. 0.5 OD units of cells were washed in $500 \mu \mathrm{L}$ of ice-cold PBS and resuspended in $50 \mu \mathrm{L}$ PBS. Next, $1.25 \mu \mathrm{L}$ of BMOE (20 mM stock in DMSO) were added and

824 the suspension was incubated for $10 \mathrm{~min}$ on ice. The reaction was quenched by addition of 1 $\mu \mathrm{L}$ of 2-mercaptoethanol (2-ME, 1.4 M stock in water). Cells were resuspended in $50 \mu \mathrm{L}$ of BPER (Thermo Fisher Scientific) containing 1 mM EDTA (pH 7.4 at RT), 14 mM 2-ME, protease inhibitor cocktail (Roche), $1 \mu \mathrm{M}$ HaloTag-TMR substrate (Promega), $0.25 \mathrm{U} / \mu \mathrm{L}$ Benzonase (Merck), and $0.1 \mathrm{U} / \mu \mathrm{L}$ ReadyLyse Lysozyme (Lucigen). The suspension was incubated for 5 min at RT and then for 10 min at $37^{\circ} \mathrm{C}$, after which $16.6 \mu \mathrm{L}$ of $4 x \mathrm{LDS}$ sample buffer containing $6 \%(\mathrm{v} / \mathrm{v}) 2-\mathrm{ME}$ were added and the sample was incubated for $5 \mathrm{~min}$ at $95^{\circ} \mathrm{C} .10 \mu \mathrm{L}$ of sample 
buffer. Gels were scanned on a Typhoon FLA9000 (GE Healthcare) with Cy3 setup.

833 Quantification of bands was performed with Wolfram Mathematica using moving median estimation of background signal. Credible intervals were estimated from posterior distributions using a normally distributed likelihood with mean $\mu$ and standard deviation $\sigma$, a uniform prior over $[0,1]$ for $\mu$ and a $1 / \sigma^{2}$ prior for $\sigma$.

839 The E. coli chromosome entrapment assay was based on protocols developed for B. subtilis

840 (Vazquez Nunez et al., 2019; Wilhelm et al., 2015). Stationary phase culture were inoculated

841 into $100 \mathrm{~mL} \mathrm{LB}$ and grown to $\mathrm{OD} 0.2-0.3$ at $22{ }^{\circ} \mathrm{C}$, which is the permissive temperature for

842 ATPase deficient mukFEB strains. We obtained similar results at $37^{\circ} \mathrm{C}$ for WT ATPase strains.

843 Cells were harvested by centrifugation, washed in $1 \mathrm{~mL}$ of ice-cold PBS, and 14 OD units were resuspended in $720 \mu \mathrm{L}$ PBS. $18 \mu \mathrm{L}$ BMOE (20 mM in DMSO) were added, and the suspension was incubated for $10 \mathrm{~min}$ on ice. The reaction was quenched by addition of $14.4 \mu \mathrm{L} 2-\mathrm{ME}(1.4$

$846 \mathrm{M}$ in water). Cells were resuspended in $200 \mu \mathrm{L}$ PBS containing $10 \mathrm{mM}$ EDTA ( $\mathrm{pH} 7.4$ at RT), 847 protease inhibitor cocktail (Roche), and $5 \mu \mathrm{M}$ HaloTag-TMR substrate (Promega) and incubated for $15 \mathrm{~min}$ at $37^{\circ} \mathrm{C}$ with shaking. Samples were protected from light from now on.

849 For input samples, 0.5 OD units from the labelling mix were collected, resuspended in $50 \mu \mathrm{L}$ B-PER (Thermo Fisher Scientific) with 1 mM EDTA (pH 7.4 at RT), protease inhibitor cocktail,

852 for $1 \mathrm{~h}$ at RT. Input samples were stored at $-20^{\circ} \mathrm{C}$ after addition of $16.6 \mu \mathrm{L} 4 \mathrm{x}$ LDS sample 853 buffer containing $6 \%(\mathrm{v} / \mathrm{v})$ 2-ME. Two agarose plugs per sample were formed each by mixing $854100 \mu \mathrm{L}$ cells with $100 \mu \mathrm{L}$ low-melt agarose $\left(2 \%(\mathrm{w} / \mathrm{v})\right.$, freshly melted at $80^{\circ} \mathrm{C}$ and equilibrated 

plugs were pooled into $3 \mathrm{~mL}$ B-PER with $10 \mathrm{mM}$ EDTA (pH 7.4 at RT), protease inhibitor cocktail, and $10 \mathrm{U} / \mu \mathrm{L}$ ReadyLyse lysozyme and incubated for $2.5 \mathrm{~h}$ at RT on a roller. Plugs were transferred into $50 \mathrm{~mL}$ TGES (25 mM Tris, $192 \mathrm{mM}$ glycine, $10 \mathrm{mM}$ EDTA (pH 7.4 at RT), $0.1 \%$ SDS) and incubated for $2 \mathrm{~h}$ at RT on a roller. Plugs were mounted in a $1 \%(\mathrm{w} / \mathrm{v})$ agarose gel in TGES and subjected to electrophoresis in TGES at $10 \mathrm{~mA} \mathrm{~cm}^{-2}$ for $1.5 \mathrm{~h}$ in a chamber cooled on ice. Next, plugs were transferred into $50 \mathrm{~mL}$ PBS, incubated for $2 \mathrm{~h}$ at RT on a roller, before transfer to $2 \mathrm{~mL}$ tubes and melting at $80^{\circ} \mathrm{C}$ for $3 \mathrm{~min}$ with occasional vortexing. The solution was incubated at $45^{\circ} \mathrm{C}$ for 5 min, thoroughly mixed with $200 \mu \mathrm{L}$ PBS containing $20 \mathrm{mM} \mathrm{MgCl} 2$ and $0.25 \mathrm{U} / \mu \mathrm{L}$ Benzonase and solidified on ice. Samples were incubated at $37^{\circ} \mathrm{C}$ for $30 \mathrm{~min}$ and stored at $-80{ }^{\circ} \mathrm{C}$ overnight. Next, samples were thawed at RT for 20 min and spun at $21,000 \times \mathrm{g}$ and $4{ }^{\circ} \mathrm{C}$ for $15 \mathrm{~min}$. Extract from both plugs was combined and passed through a 
bioRxiv preprint doi: https://doi.org/10.1101/2021.06.29.450292; this version posted June 29, 2021. The copyright holder for this preprint

(which was not certified by peer review) is the author/funder, who has granted bioRxiv a license to display the preprint in perpetuity. It is made available under aCC-BY-NC-ND 4.0 International license.

877 Quantification of bands was performed with Wolfram Mathematica using moving median

878 estimation of background signal. Credible intervals were estimated from posterior

879 distributions using a normally distributed likelihood with mean $\mu$ and standard deviation $\sigma$, a

880 uniform prior over $[-10 \mu, 10 \mu]$ for $\mu$ and a $1 / \sigma^{2}$ prior for $\sigma$.

881 


\section{Acknowledgements}

883 We thank J. Prince, G. Fisher and D. Sherratt (Oxford, UK) for discussions and sharing of

884 unpublished results; R. Vazquez Nuñez and S. Gruber (Lausanne, Switzerland) for discussions

885 and advice on the chromosome entrapment assay; all members of the Löwe and K. Nasmyth

886 (Oxford, UK) groups for discussions; G. Cannone and all members of the LMB electron

887 microscopy facility for excellent EM training and support; T. Darling and J. Grimmett (LMB

888 scientific computing) for computing support. F.B. was supported by an EMBO Advanced

889 fellowship (ALTF 605-2019).

890

891 Author contributions

892 F.B. performed protein purification, cryo-EM sample preparation, cryo-EM data acquisition

893 and analysis, model building, strain construction and all other experiments; L.F.H.F. designed

894 the genome mutagenesis strategy; J.W.C. supervised technology development; J.L.

895 supervised the overall study; F.B. prepared the manuscript with contributions from all

896 authors.

897

\section{Competing interests}

899 The authors declare no competing interests. 
bioRxiv preprint doi: https://doi.org/10.1101/2021.06.29.450292; this version posted June 29, 2021. The copyright holder for this preprint

(which was not certified by peer review) is the author/funder, who has granted bioRxiv a license to display the preprint in perpetuity. It is made available under aCC-BY-NC-ND 4.0 International license.

A
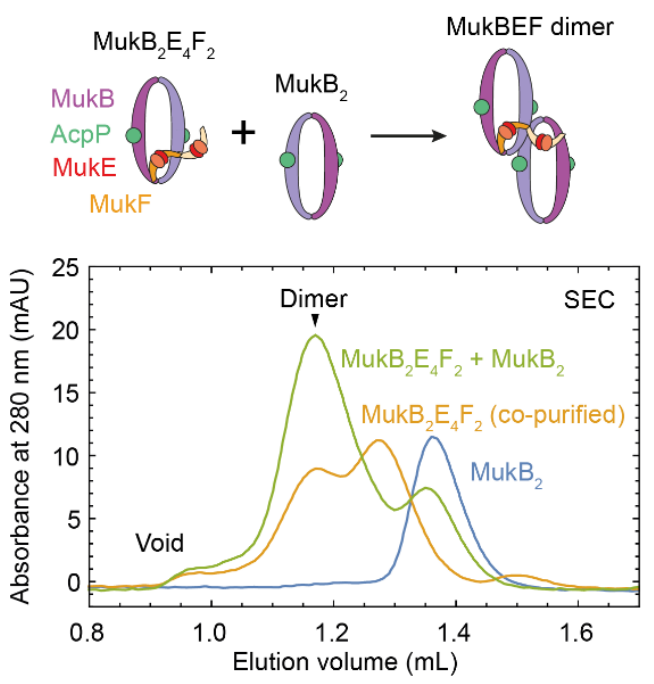

E
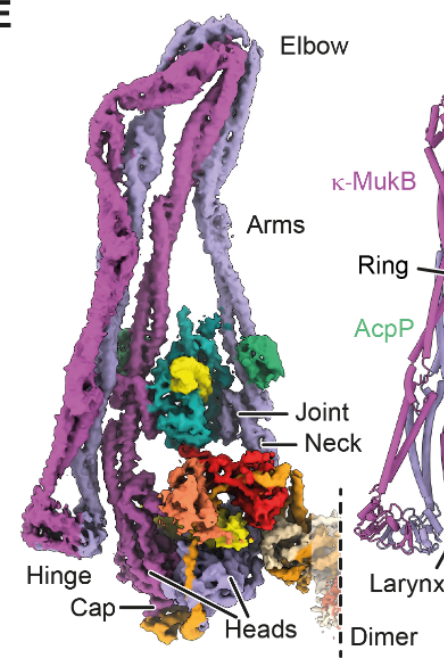

G

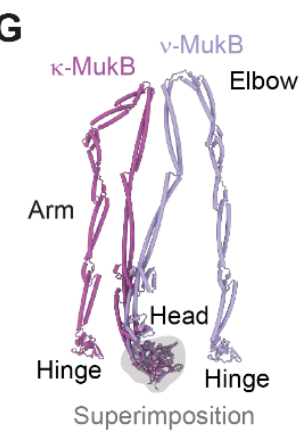

902
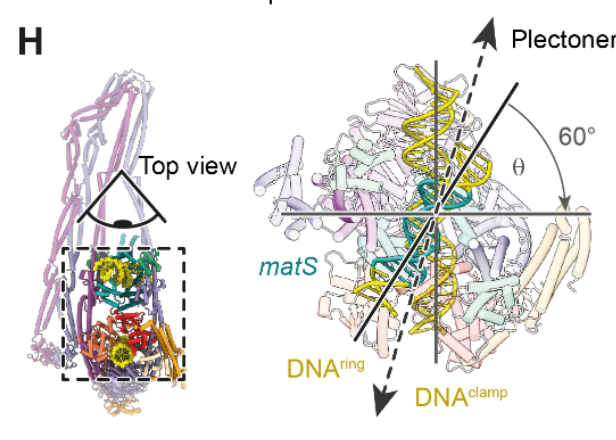

cWHD

B MukBEOEF dimer
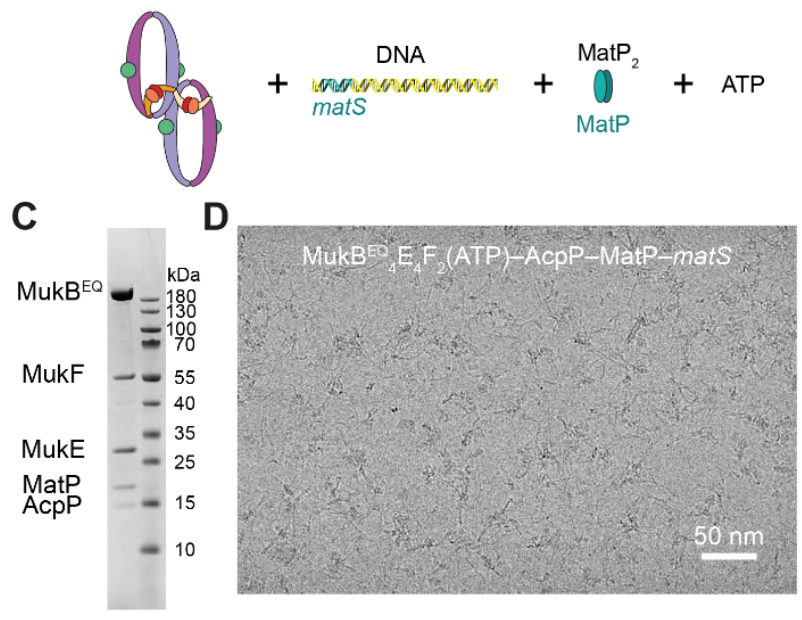

$\mathbf{F}$

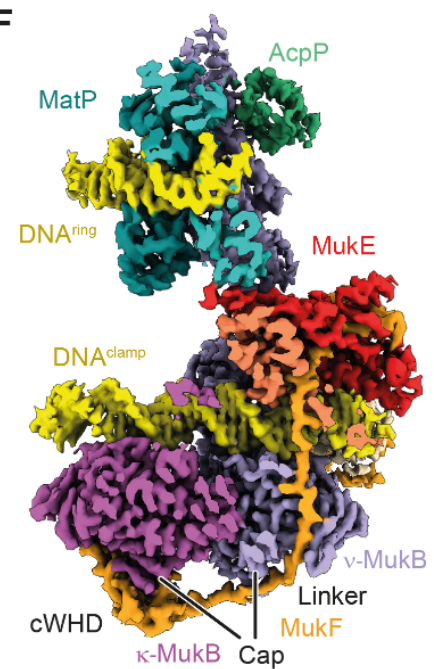

axis

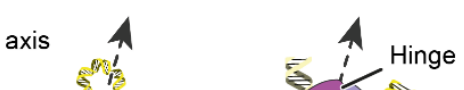

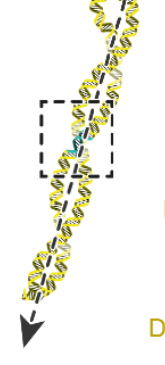

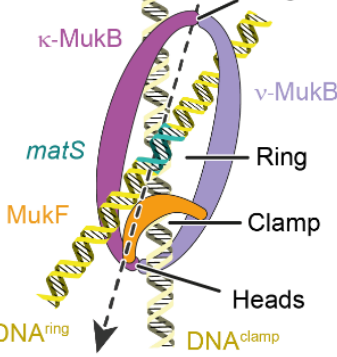

simplified topology

Figure 1. Cryo-EM structure of MukBEF-MatP bound to two distinct DNA double helices. 
904 (A) Reconstitution of MukBEF dimers. Co-purified MukBEF and free MukB were mixed (top)

905 and resolved by SEC (bottom). (B) Composition of the MukBEF-MatP-matS sample used for

906 structure determination. (C) Coomassie stained SDS-PAGE gel of the reconstituted complex

907 used for cryo-EM. (D) Example micrograph of the sample used for structure determination.

908 (E) 4.6 Å resolution cryo-EM density map (left) and complete atomic model (middle, right) of

909 the DNA bound MukBEF-MatP monomer. (F) Slice through a $3.1 \AA$ A resolution cryo-EM density

910 map of the DNA binding region of MukBEF-MatP. (G) $\kappa-M u k B$ and $v-M u k B$ superimposed on

911 the head domain. The arms adopt radically different conformations. (H) DNA binding topology

912 on plectonemic loops inferred from the DNA crossing angle. The schematic on the right shows

913 the simplified topology used for clarity throughout, with the in-reality elbow folded

914 conformation flattened into a ring. See also Figures S1, S2 and S3. 


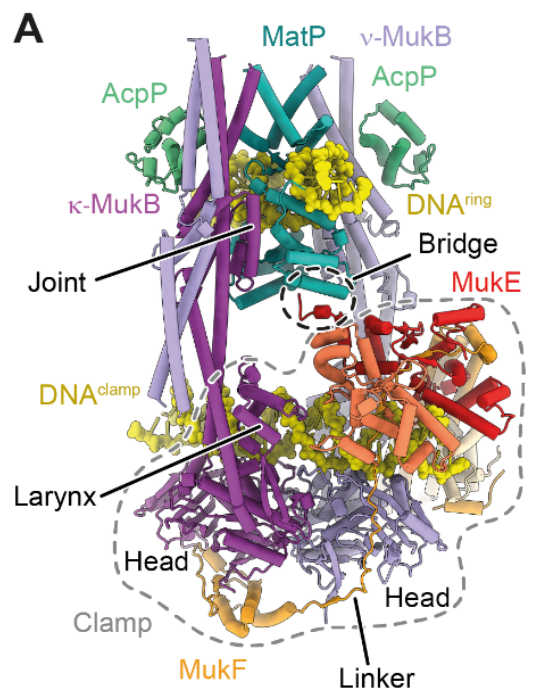

E

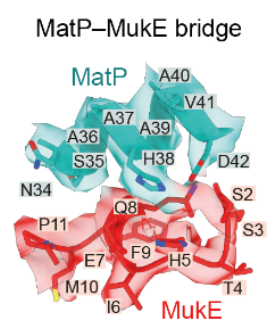

$\mathbf{F}$

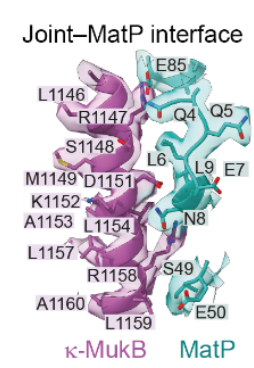

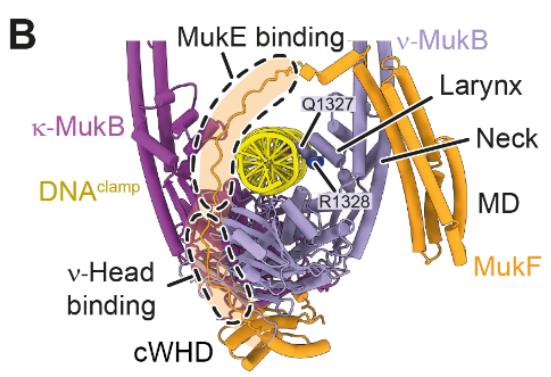

C MukF-DNA interface $\mathbf{D}_{\text {Head-DNA interface }}$

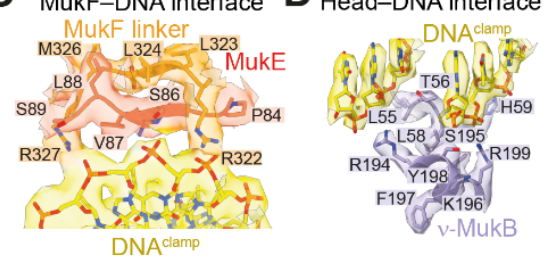

G

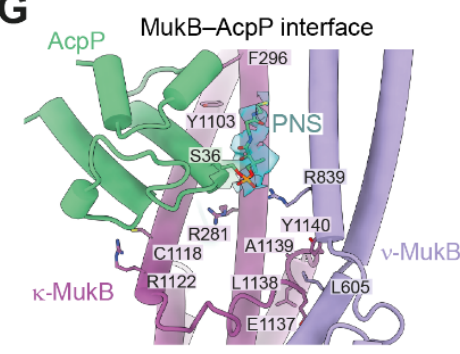

917 Figure 2. DNA binding and subunit interfaces of the MukBEF-MatP complex.

918 (A) Model of the DNA-bound head module. (B) Path of the kleisin MukF and DNA contacts of

919 the MukB larynx. (C) Interface between the MukF linker and the clamped DNA. (D) Interface

921 MukE and MatP. (F) Interface between the MukB joint and MatP. (G) Interfaces between

922 MukB and AcpP, and between the к-MukB joint and the hinge-proximal arm of $v$-MukB. See

923 also Figures S2 and S3. 
A

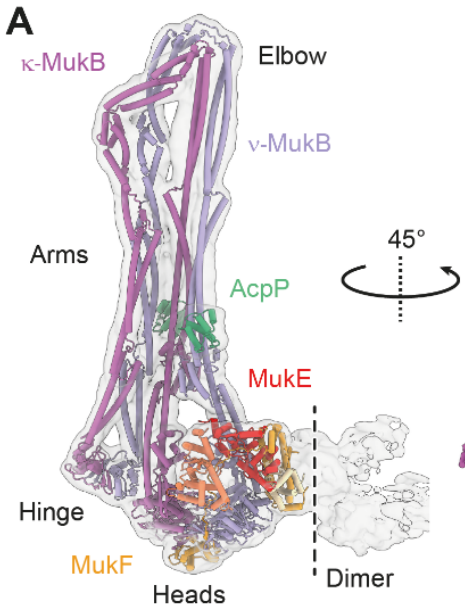

C

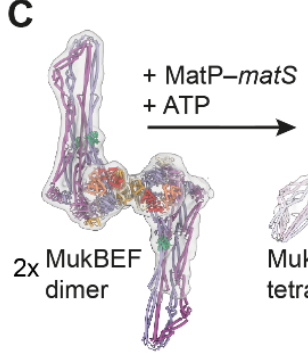

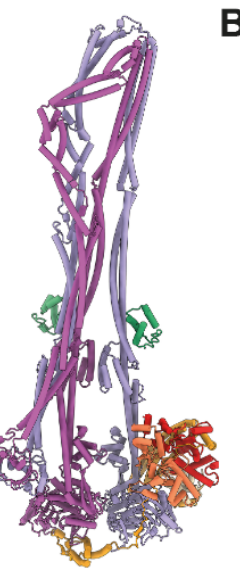

Dimer 1 Dimer 2

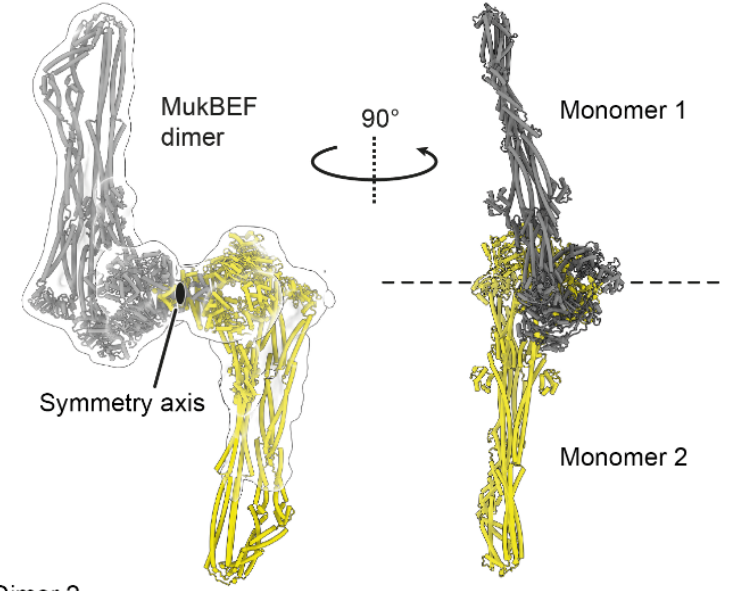

$\longrightarrow$
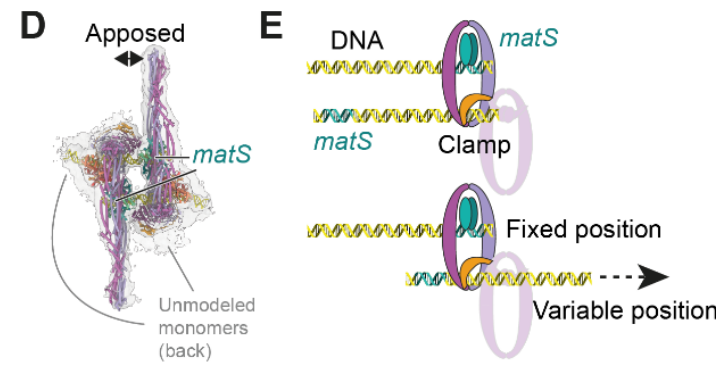

926 Figure 3. Architecture of apo-MukBEF and the MukBEF dimer.

(A) Model of the apo-MukBEF monomer and 6.8 Å cryo-EM density at low contour level. (B)

928 Model for the apo-MukBEF dimer and $13 \AA$ cryo-EM density. (C) $11 \AA \AA$ cryo-EM density and model for two MukBEF dimers bridged by four MatP-DNA complexes ('MukBEF tetrad'). The apo-MukBEF dimer is shown on the left. (D) $11 \AA ̊$ cryo-EM density and model for a MukBEF

931 tetrad with closely apposed dimers. Only one monomer for each MukBEF dimer was modelled due to weak density for their partner monomers. (E) Schematic for variable positioning of the

933 clamp DNA binding site, as shown in C and D. Only a single MukBEF dimer and only two of the four DNAs are shown for clarity. Related to Figures S2 and S3. 


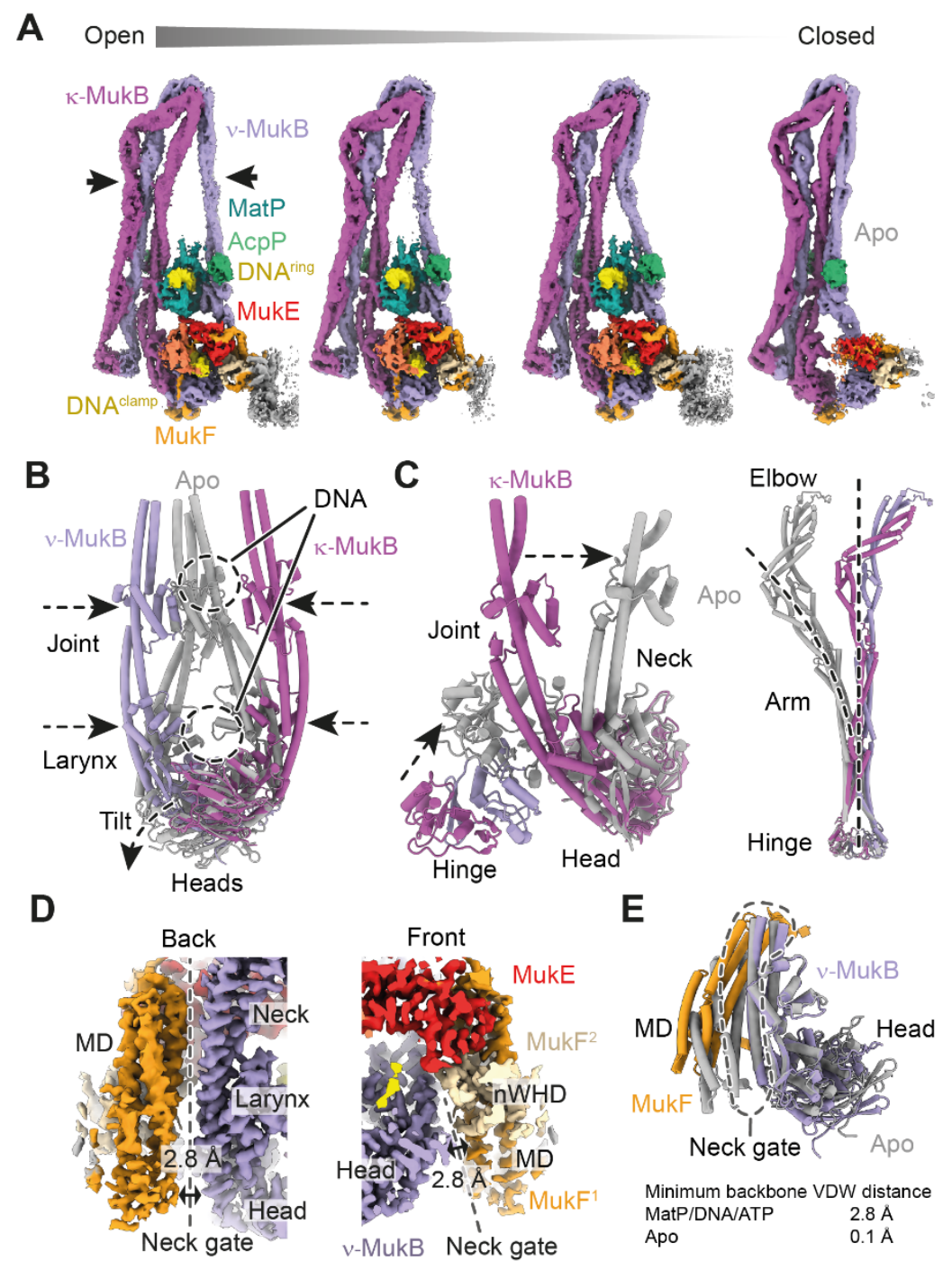

937 Figure 4. Conformational changes associated with release of MatP/DNA/ATP.

938 (A) Cryo-EM densities for the MukBEF-MatP-DNA complexes with different arm

939 conformations. Density of apo-MukBEF with fully closed arms is shown on the right. (B)

940 Blocking of MatP and DNA binding sites at the MukB joint and larynx. Structures were

941 superimposed on the ATPase domains. MatP/DNA/ATP-bound conformation is shown in

942 color, apo conformation in grey. (C) Conformational change at the MukB neck/hinge interface

943 (left) and at the hinge-proximal arm (right). Structures were superimposed on the ATPase

944 domain (left) or the hinge (right). (D) Cryo-EM density at the neck gate in the MatP/DNA/ATP 
945 bound state. The solvent accessible cleft between MukB and MukF is indicated by a double

946 arrow. (E) Superimposition of the neck gate in apo and MatP/DNA/ATP bound states (top).

947 Minimum backbone VDW distances of the interface are given (bottom). See also Figures S2

948 and S3.

949 
A

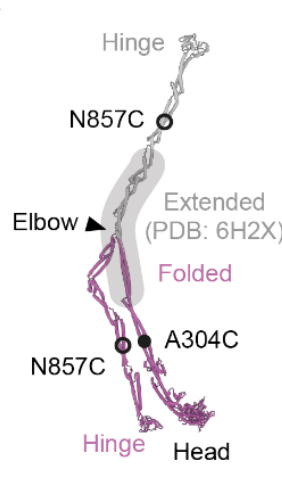

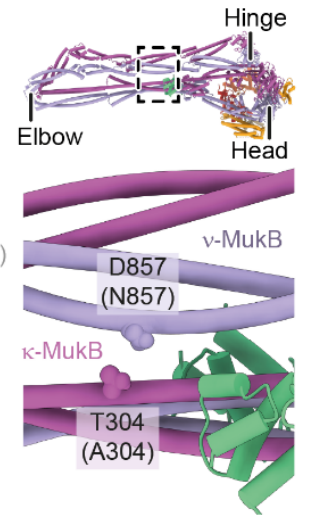

B
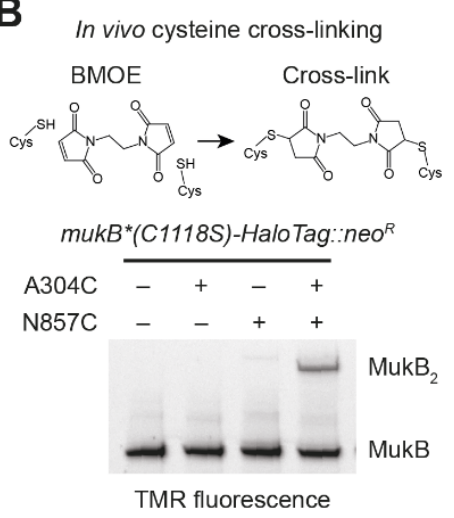

950

951 Figure 5. Detection of arm folding in vivo.

952 (A) Residues employed as sensors for the folded conformation. The folded conformation and

953 a tentative extended conformation based on the structure of the extended elbow (PDB: 6H2X)

954 are shown on the left. A close-up on the $P$. thracensis structure is shown on the right.

955 Corresponding E. coli residues are in parentheses. (B) BMOE reaction scheme (top) and BMOE

956 mediated in vivo cysteine cross-linking of $E$. coli strains carrying sensor cysteine mutations

957 (bottom). Reaction products were detected by SDS-PAGE and in-gel fluorescence using a TMR

958 fluorophore bound to MukB-HaloTag. See also Figures S4 and S5.

959

960 
bioRxiv preprint doi: https://doi.org/10.1101/2021.06.29.450292; this version posted June 29, 2021. The copyright holder for this preprint (which was not certified by peer review) is the author/funder, who has granted bioRxiv a license to display the preprint in perpetuity. It is made available under aCC-BY-NC-ND 4.0 International license.

A

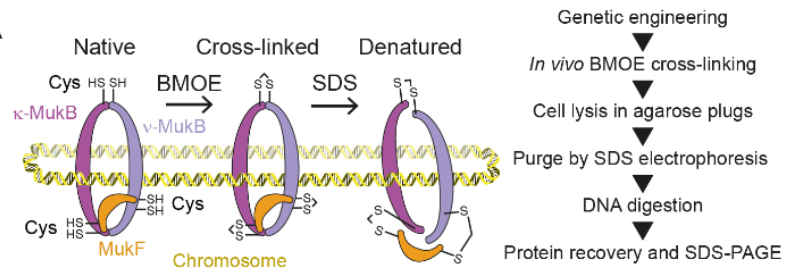

C
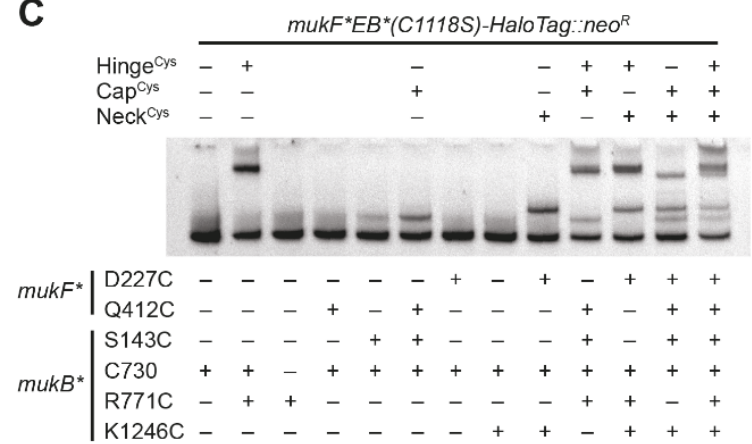

D

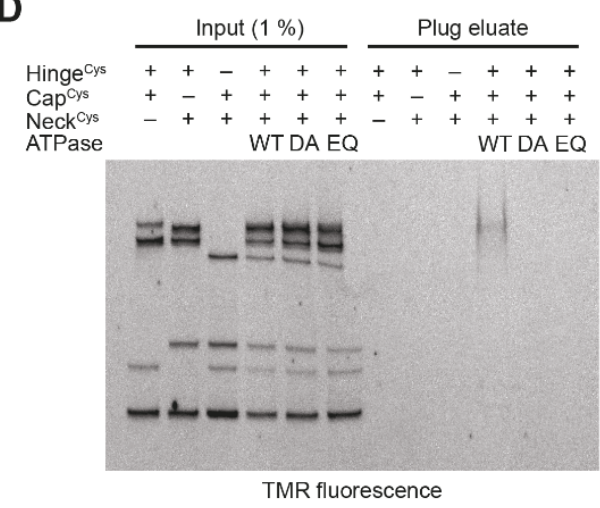

$\mathbf{F}$

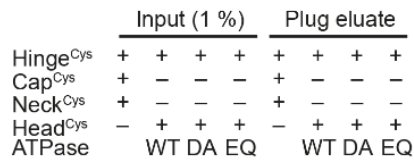

961

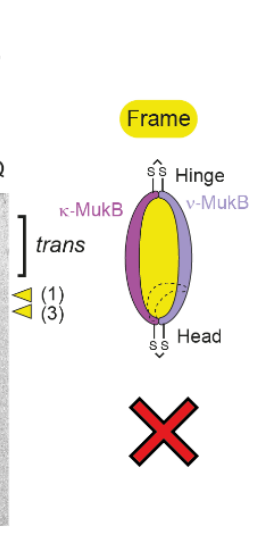

B

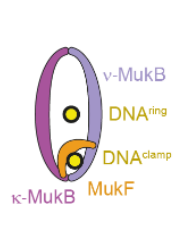

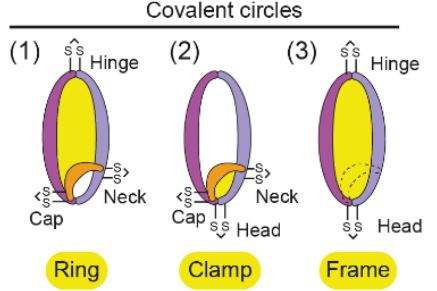

mukF*E$B^{*}(C 1118 S)$-HaloTag::neo ${ }^{R} \quad m u k F^{*} E B^{*}(C 1118 S)$-HaloTag::neo
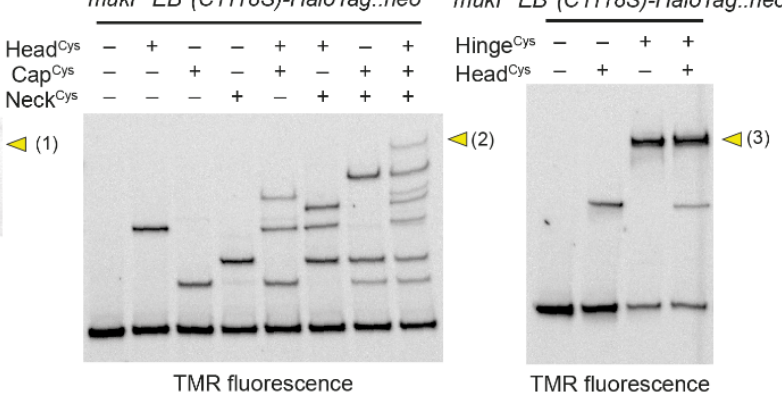

E

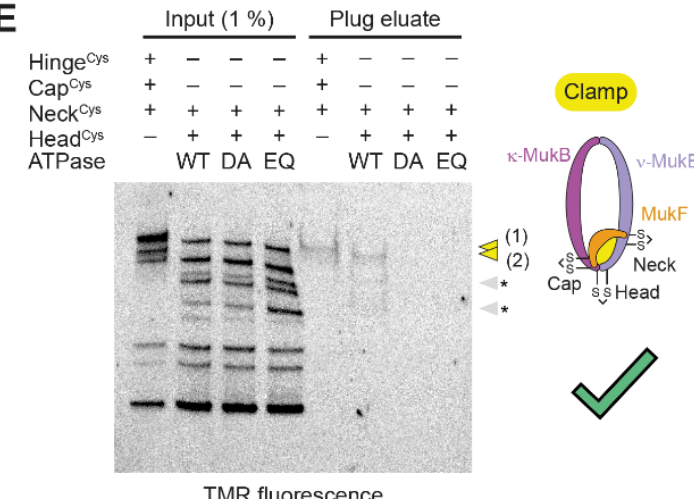

G

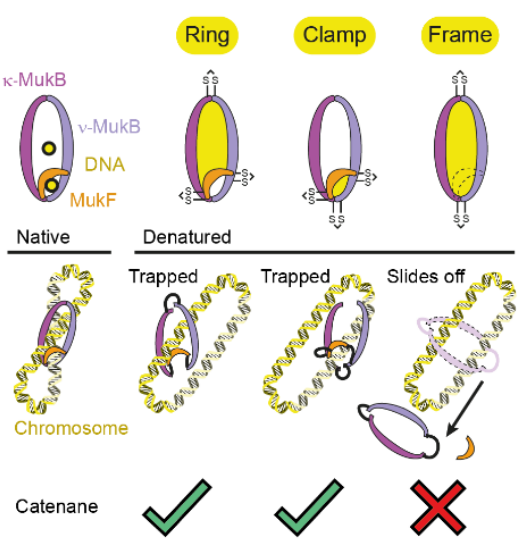

H

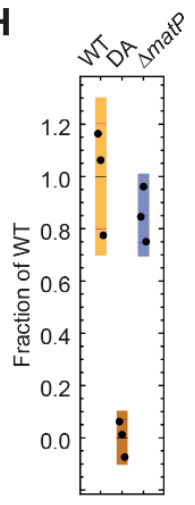

Figure 6. Mapping of DNA binding topology in vivo.

963 (A) Principles (left) and workflow (right) of the chromosome entrapment assay in agarose plugs. (B) Combinations of cross-links used for probing DNA entrapment in ring, clamp and frame compartments. Hinge cross-link: C730 and R771C in MukB; cap cross-link: Q412C in 
MukF and S143C in MukB; neck cross-link: D227C in MukF and K1243C in MukB; head cross-

967 link: G67C in MukB (Figures S5A and S6A). (C) Combinatorial cross-linking for identification of reaction species. Combinations: Hinge, cap, and neck cross-links (left); cap, neck, and head

969 cross-links (middle); head and hinge (right). C730 was mutated to serine when indicated by '-

970 '. Cells were grown to stationary phase. Detection as in Figure 5B. (D) Chromosome

971 entrapment in MukBEF with covalently closed ring compartment. Input and agarose plug

972 eluate are shown. Detection as C. The circular species is retained only in WT ATPase cells. DA,

973 D1406A (blocks ATP binding); EQ, E1407Q (blocks ATP hydrolysis). ATPase is WT if not

974 indicated otherwise. (E) Chromosome entrapment in MukBEF with covalently closed clamp

975 compartment. As in D. Species that have undergone chemical cross-link reversal during

976 sample preparation are marked with asterisks. (F) Chromosome entrapment in MukB with

977 covalently closed frame compartment. As in D. Species produced by higher oligomers in EQ

978 mutants (trans cross-links) are indicated. (G) Structure-based topological interpretation of the

979 entrapment reactions. Only the frame species can slide off DNA, because it does not form a

980 protein/DNA catenane. $(\mathbf{H})$ Chromosome entrapment in the ring in the absence of MatP.

981 Entrapment signal relative to WT is shown for biological triplicates. Black lines indicate means,

982 purple lines indicate standard deviations, and colored bars indicate $95 \%$ credible intervals.

983 See also Figures S5 and S6. 
A

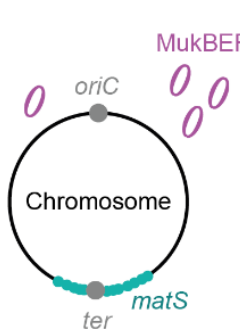

B

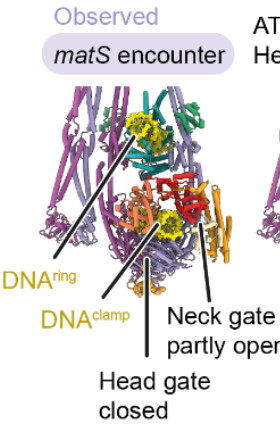

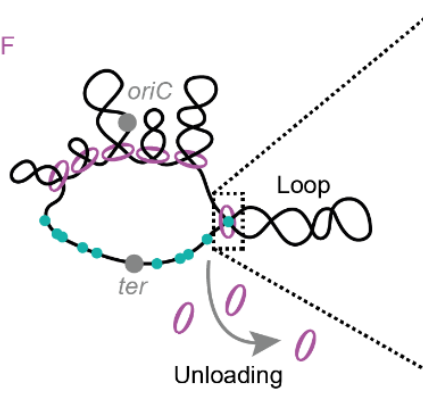

Unloading

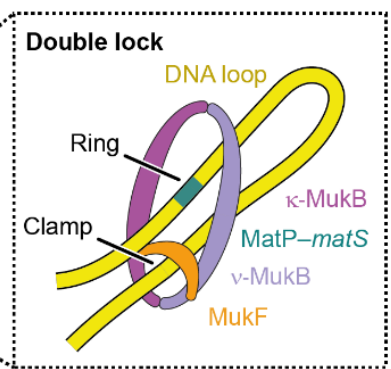

TP hydrolysis Head disengagement Arm zip-up

DNA dissociation

Observed

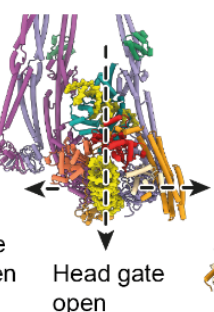
open
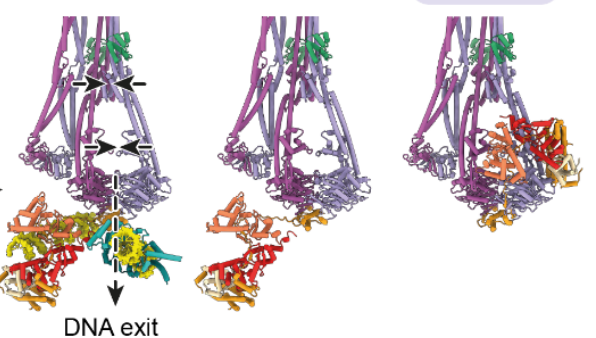

through neck and head gates

Figure 7. Model for DNA binding and unloading at matS sites.

(A) Schematic for association of MukBEF with MatP-matS in the Ter macrodomain. MukBEF and unloads via the double-lock topology in the context of a plectonemic loop. (B) Model for unloading of DNA. A MatP-matS encounter is followed by ATP hydrolysis and opening of the head gate to permit exit of the clamped DNA. matS DNA follows through neck and head gates,

993 facilitated by the bridge between MatP and MukE. Arm zip-up prevents reversal, and the neck purple. 
A

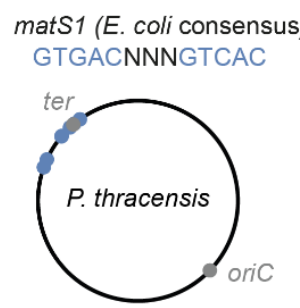

B

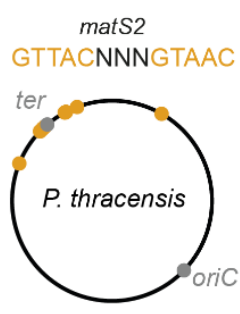

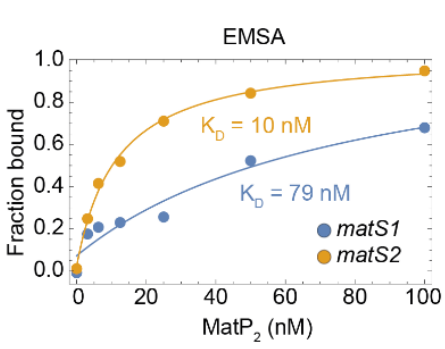

C

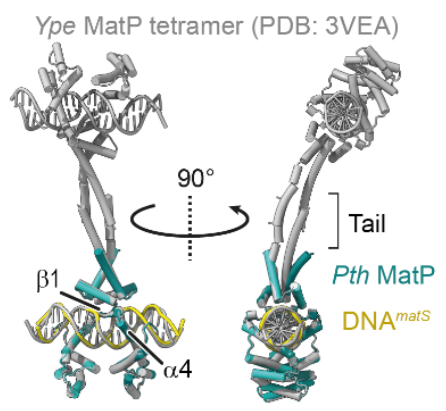

998 Figure S1. matS binding by P. thracensis MatP. Related to Figure 1.

999 (A) Location of the E. coli matS consensus sequence (left) and the matS sequence used for structure determination (right) mapped onto the P. thracensis chromosome. (B) Affinities of P. thracensis MatP for matS sites shown in A as determined by EMSA. (C) Superimposition of MatP-matS in the MukBEF-bound form (colored) and a crystal structure in the absence of MukBEF (gray, PDB: 3VEA). Positions of the matS binding elements $\alpha 1$ and $\beta 1$ and the Cterminal tetramerization tail are indicated. Ype, Yersinia pestis; Pth, P. thracensis. 
bioRxiv preprint doi: https://doi.org/10.1101/2021.06.29.450292; this version posted June 29, 2021. The copyright holder for this preprint (which was not certified by peer review) is the author/funder, who has granted bioRxiv a license to display the preprint in perpetuity. It is made available under aCC-BY-NC-ND 4.0 International license.

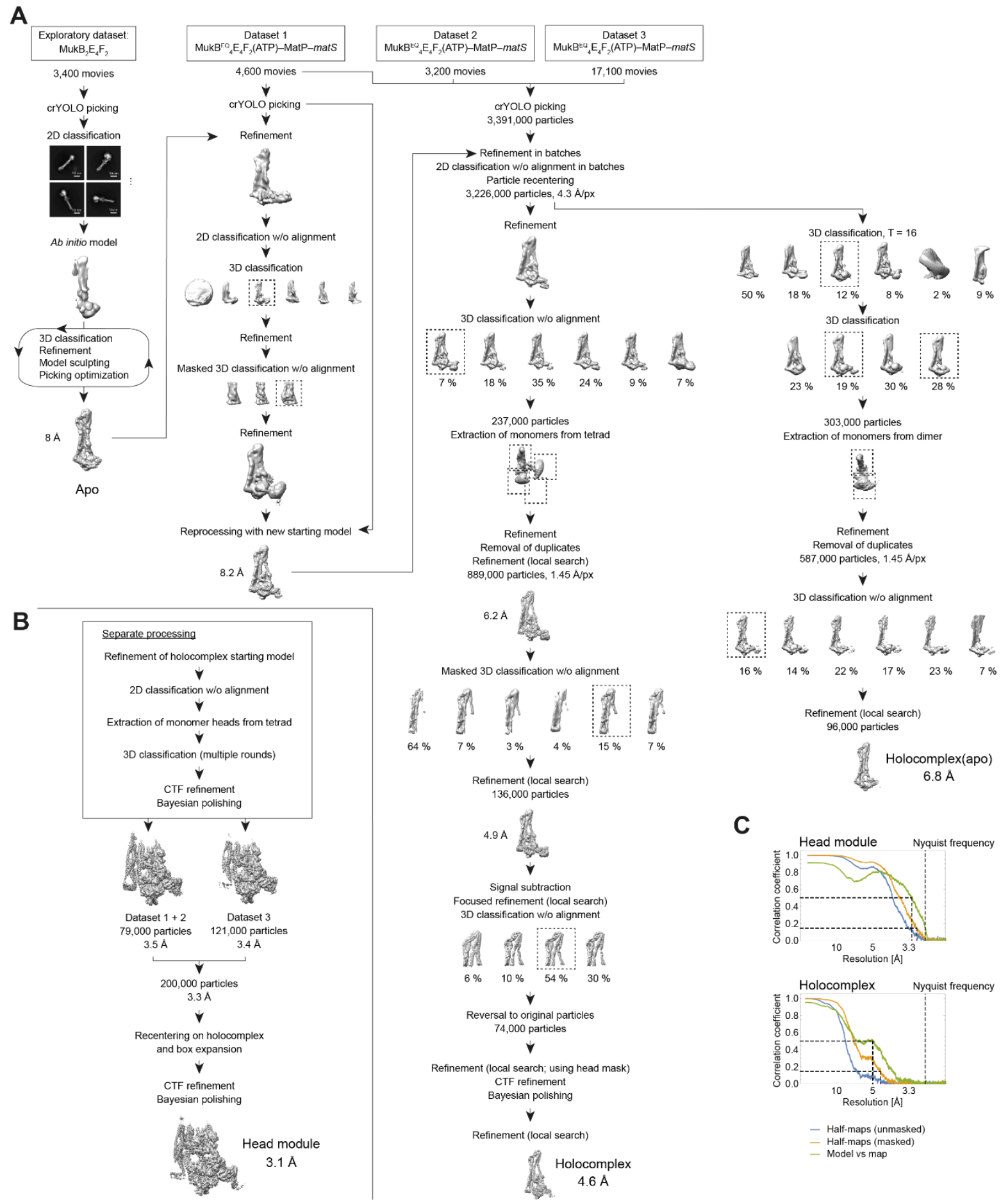

1007 Figure S2. Cryo-EM data analysis workflow. Related to Figures 1-3.

1008 (A) The processing tree for structure determination of MukBEF-MatP-DNA and apo-MukBEF monomers. (B) Data processing tree for focused structure determination of the head module.

1010 (C) FSC curves for MukBEF-MatP-DNA head module and holocomplex structures. 
A

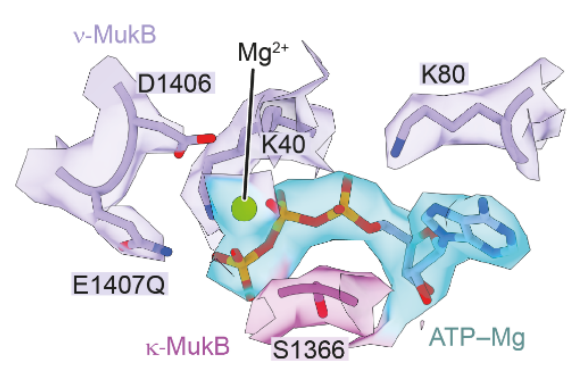

C

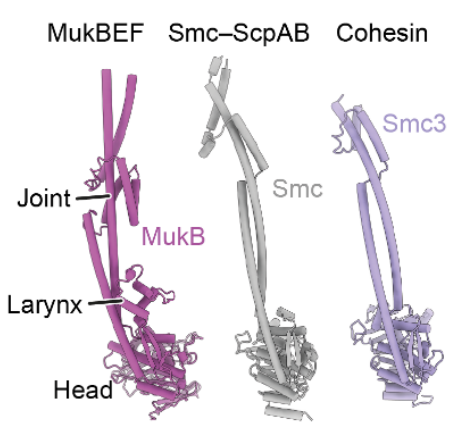

E

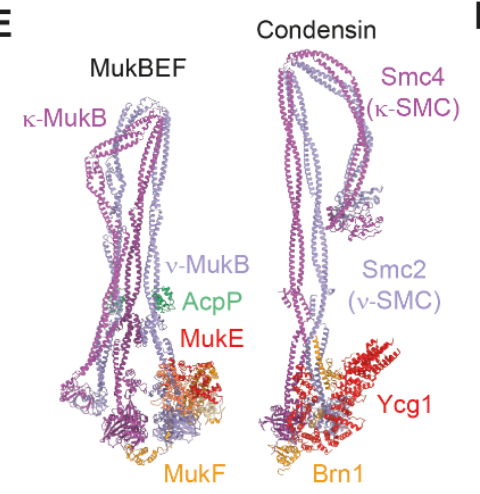

D
B

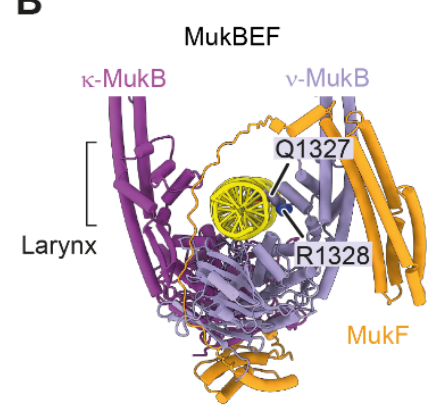

Cohesin

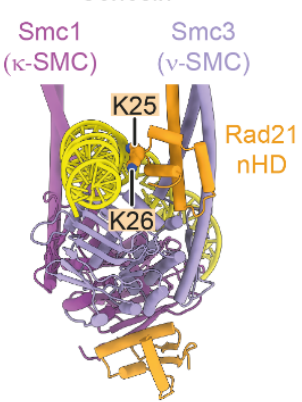

$\begin{array}{cc}\mathrm{Smc1} & \mathrm{Smc3} \\ (\mathrm{K}-\mathrm{SMC}) & (\mathrm{v}-\mathrm{SMC})\end{array}$

MukBEF

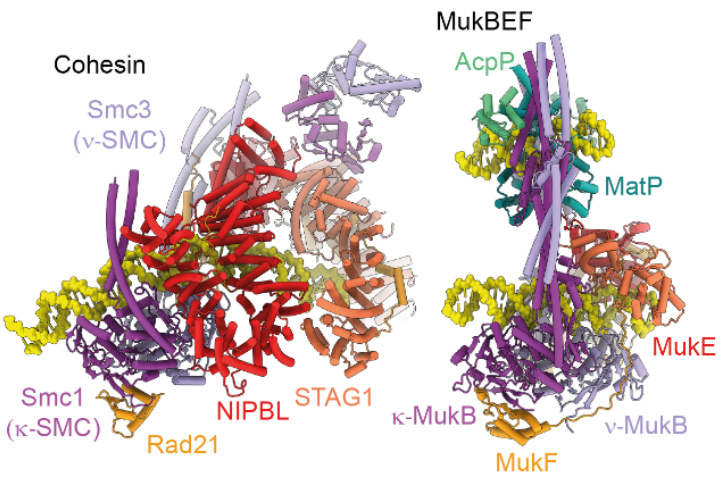

G
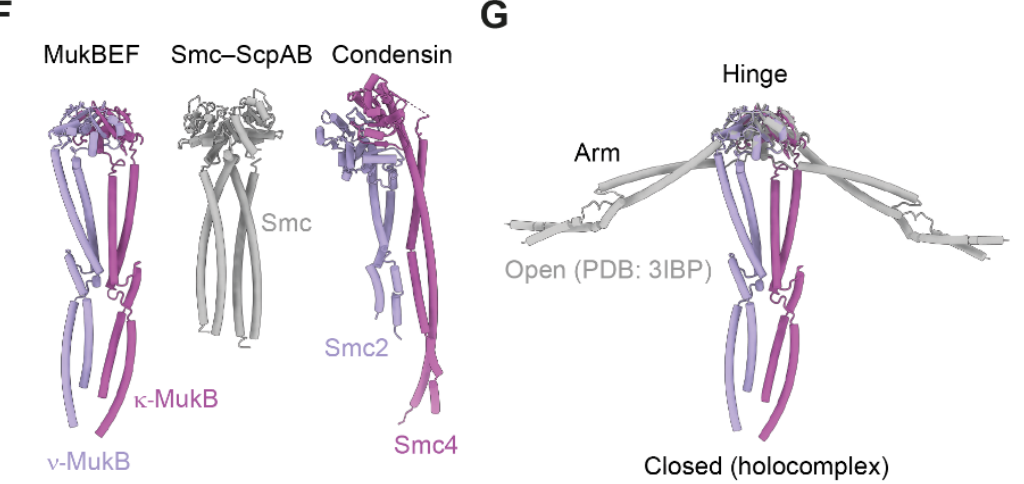

1013 Figure S3. Conserved structural features and comparison with other SMC complexes. Related

1015 (A) Cryo-EM density and atomic model for the nucleotide binding site at the $v$-MukB Walker

1016 A and B motives and the $\kappa-M u k B$ signature motive. (B) Architecture of the neck gate in 1017 MukBEF (left) and cohesin (right, PDB: 6WG3). An asymmetric DNA contact at the larynx of 1018 MukBEF and the N-terminal helical domain of Rad21 is indicated. (C) Comparison of the head1019 proximal regions of MukB, Smc (PDB: 5XEI) and Smc3 (PDB: 6WGE). (D) Architecture of SMC- 
1020 DNA clamps. Models were aligned on the $\kappa$-SMC ATPase domain. PDB: 6S85, 6WG3. (E)

1021 Comparison of apo-MukBEF and apo-condensin (PDB: 6YVU). (F) Comparison of the hingeproximal regions of MukB, Smc (PDB: 4RSJ) and Smc2/4 (PDB: 4RSI). (G) Superimposition of

1023 the hinge-proximal region of MukB in an open conformation (PDB: 3IBP) and in the closed conformation. 


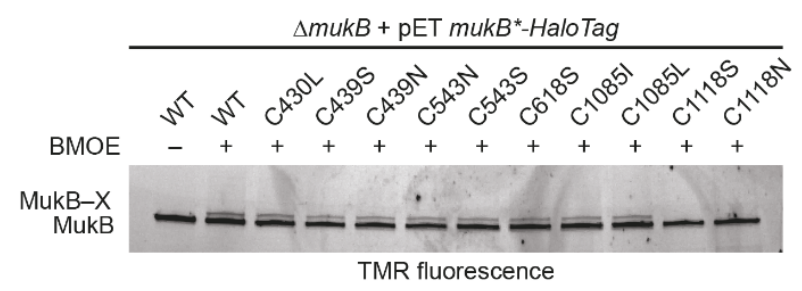

1027 Figure S4. Background cross-linking of endogenous MukB cysteines. Related to Figure 5.

1028 Screening of endogenous cysteines for background cross-linking. E. coli $\Delta$ mukB was

1029 transformed with plasmids carrying mukB-HaloTag variants under control of a T7 promoter.

1030 Leaky expression in the strain lacking a T7 RNA polymerase gene produced about $40 \%$ MukB-

1031 HaloTag compared to mukB-HaloTag expressed from the endogenous locus. The mukB null

1032 phenotype was complemented in all cases. Cells were treated with BMOE and proteins were

1033 detected by in-gel fluorescence. C1118S and C1118N abolished background cross-linking with

1034 a low-molecular weight protein. 
A
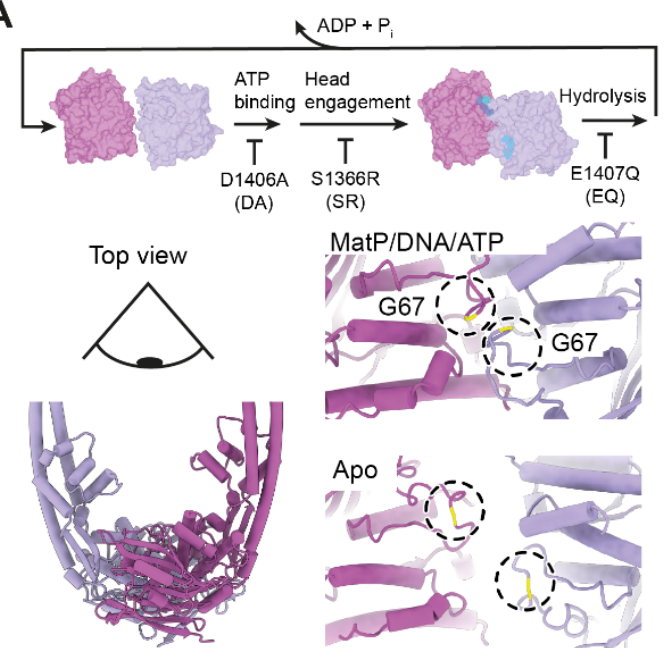

MatP/DNA/ATP
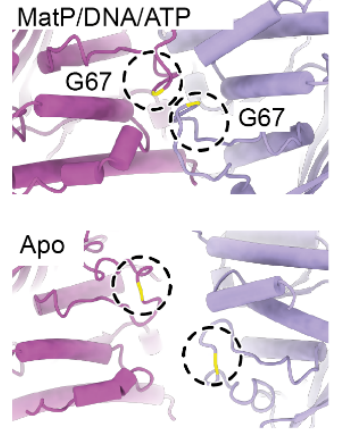

B

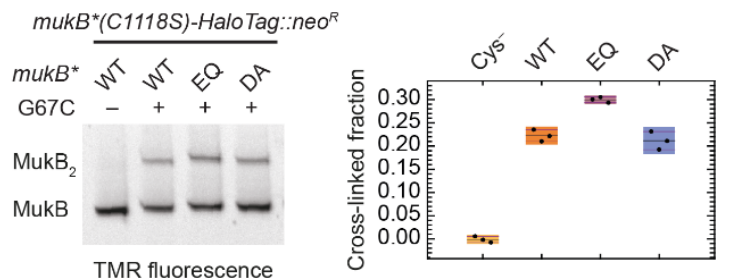

C

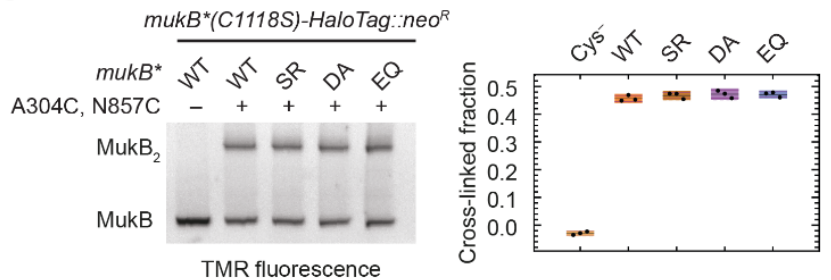

1037 Figure S5. In vivo head engagement and arm folding in ATPase mutants. Related to Figures 5

1038 and 6.

1039 (A) Schematic of the ATP hydrolysis cycle and blocking mutations (top). Location of the head 1040 engagement sensor residue G67 in MatP/DNA/ATP and apo states (bottom). S1366R ('SR')

1041 blocks head engagement, D1406A ('DA') blocks ATP binding, E1407Q ('EQ') blocks ATP

1042 hydrolysis. (B) BMOE cross-linking of strains carrying the head engagement sensor mutation

1043 G67C and ATPase blocking mutations. Cells were grown in LB for $1.5 \mathrm{~h}$ at $37^{\circ} \mathrm{C}$ before cross-

1044 linking. In-gel fluorescence is shown on the left, and quantification of three technical

1045 replicates is shown on the right. Black lines indicate means, purple lines indicate standard

1046 deviations, and colored bars indicate $95 \%$ credible intervals. (C) BMOE cross-linking of arm

1047 folding sensor strains carrying ATPase mutations. As in B. 
A

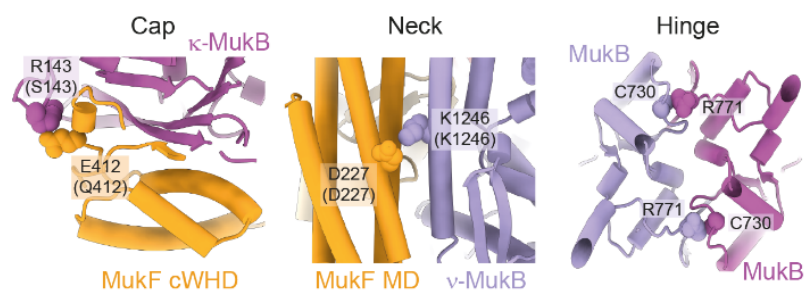

B

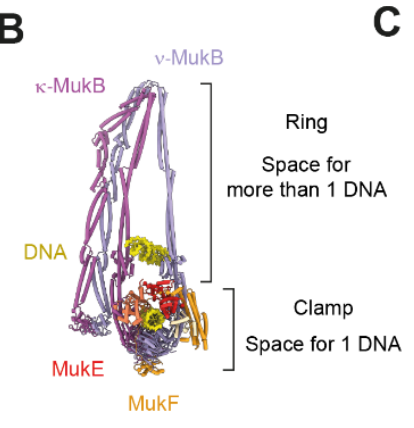

C Ring Clamp Frame

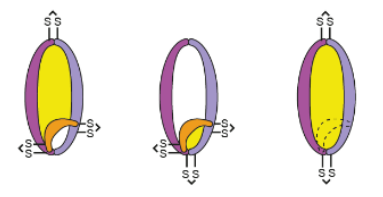

Experiment

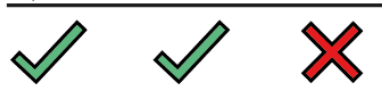

Expectation
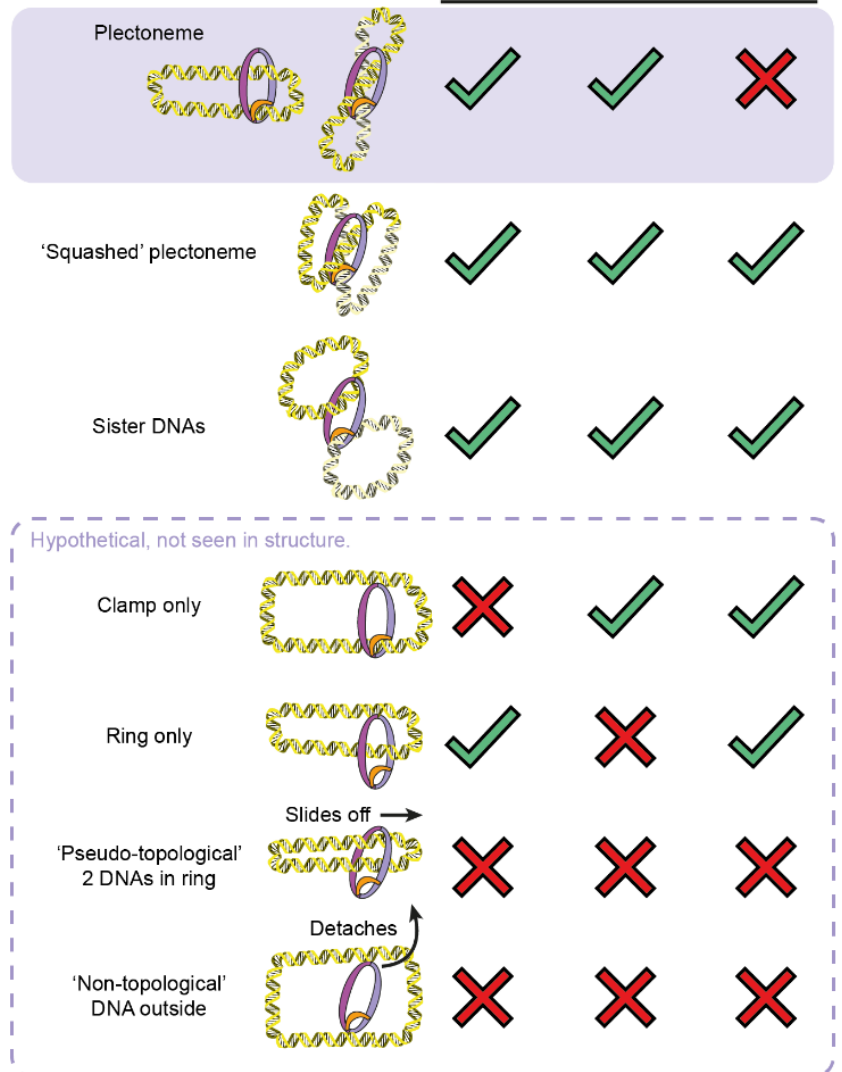

1050 Figure S6. Chromosome entrapment by MukBEF. Related to Figure 6.

1051 (A) Design of cap, neck and hinge cross-links. Location of residues chosen for cysteine mutagenesis at the hinge (left, PDB: 3IBP), cap (middle), and neck (right) is shown. Labels for 

in parentheses. (B) Space for hypothetical accommodation of additional DNA double strands.

1055 Only the ring compartment is large enough to embrace more than one DNA. (C) Comparison

1056 of experimentally observed and expected catenanes for different DNA binding topologies.

1057 The topology consistent with the experimental data is highlighted. For clarity, the double-

1058 locked plectoneme topology is also shown as a simplified version without DNA crossings.

1059 Hypothetical 'pseudo-topological' and 'non-topological' DNA loops do not produce

1060 catenanes. Their tentative formation in addition to catenated forms is therefore not excluded

1061 by the data. 
bioRxiv preprint doi: https://doi.org/10.1101/2021.06.29.450292; this version posted June 29, 2021. The copyright holder for this preprint

(which was not certified by peer review) is the author/funder, who has granted bioRxiv a license to display the preprint in perpetuity. It is made available under aCC-BY-NC-ND 4.0 International license.

1064 Table S1. Cryo-EM data collection and model statistics.

\begin{tabular}{|c|c|c|c|c|c|c|c|c|}
\hline & Head module & Holocomplex & $\begin{array}{l}\text { Holocomplex } \\
\text { (apo) }\end{array}$ & $\begin{array}{l}\text { Holocomplex } \\
\text { (partially open) }\end{array}$ & $\begin{array}{l}\text { Holocomplex } \\
\text { (open) }\end{array}$ & Tetrad & $\begin{array}{l}\text { Tetrad } \\
\text { (apposed) }\end{array}$ & $\begin{array}{l}\text { Dimer } \\
\text { (apo) }\end{array}$ \\
\hline & EMD-12656 & EMD-12657 & EMD-12658 & EMD-12658 & EMD-12658 & EMD-12662 & EMD-12663 & EMD-12664 \\
\hline & PDB 7NYW & PDB 7NYX & PDB 7NYY & PDB 7NYZ & PDB 7NZO & PDB 7NZ2 & PDB 7NZ3 & PDB 7NZ4 \\
\hline \multicolumn{9}{|l|}{ Data collection and processing } \\
\hline Magnification & 81,000 & & & & & & & \\
\hline Voltage (kV) & 300 & & & & & & & \\
\hline Electron fluence $\left(\mathrm{e}-/ \AA^{2}\right)$ & 40 & & & & & & & \\
\hline Defocus range $(\mu \mathrm{m})$ & -1 to -3 & & & & & & & \\
\hline Pixel size $(\AA)$ & 1.07 & & & & & & & \\
\hline Symmetry imposed & $\mathrm{C} 1$ & & & & & & & \\
\hline Initial particle images (no.) & $3,391,688$ & & & & & & & \\
\hline Final particle images (no.) & 200,438 & 74,064 & 96,150 & 41,109 & 60,245 & 12,010 & 8,561 & 4,197 \\
\hline Map resolution $(\AA \stackrel{\AA}{)})$ & 3.1 & 4.6 & 6.8 & 6.5 & 6.3 & 11 & 11 & 13 \\
\hline FSC threshold & 0.143 & 0.143 & 0.143 & 0.143 & 0.143 & 0.143 & 0.143 & 0.143 \\
\hline \multicolumn{9}{|l|}{ Model } \\
\hline $\begin{array}{l}\text { Initial model used } \\
\text { (PDB code) }\end{array}$ & $\begin{array}{l}\text { 3EUJ, 3EUH, } \\
\text { 3VEA, 3IBP, } \\
6 \mathrm{DFL}\end{array}$ & $\begin{array}{l}\text { Head module, } \\
6 \mathrm{H} 2 \mathrm{X}\end{array}$ & Holocomplex & Holocomplex & Holocomplex & Holocomplex & Holocomplex & $\begin{array}{l}\text { Holocomplex } \\
\text { (apo) }\end{array}$ \\
\hline Model resolution (Å) & 3.25 & 5.0 & 7.5 & 8.1 & 7.3 & - & - & - \\
\hline FSC threshold & 0.5 & 0.5 & 0.5 & 0.5 & 0.5 & & & \\
\hline Map sharpening B factor ( $(\AA 2)$ & -33 & -87 & -174 & -162 & -157 & - & - & - \\
\hline \multicolumn{9}{|l|}{ Model composition } \\
\hline Non-hydrogen atoms & 24,792 & 36,100 & 31,563 & 36,100 & 36,100 & 148,563 & 74,338 & 63,192 \\
\hline Protein residues & 2,795 & 4,186 & 3,910 & 4,186 & 4,186 & 16,752 & 8372 & 7824 \\
\hline Nucleic acid residues & 104 & 104 & - & 104 & 104 & 614 & 312 & - \\
\hline \multirow[t]{3}{*}{ Ligands } & PNS: 2 & PNS: 2 & PNS: 2 & PNS: 2 & PNS: 2 & PNS: 8 & PNS: 4 & PNS: 4 \\
\hline & ATP: 2 & ATP: 2 & & ATP: 2 & ATP: 2 & ATP: 8 & ATP: 4 & \\
\hline & Mg: 2 & Mg: 2 & & Mg: 2 & Mg: 2 & Mg: 8 & Mg: 4 & \\
\hline \multicolumn{9}{|l|}{ R.m.s. deviations } \\
\hline Bond lengths (Å) & 0.004 & 0.004 & 0.004 & 0.009 & 0.005 & 0.004 & 0.004 & 0.005 \\
\hline Bond angles $\left({ }^{\circ}\right)$ & 0.867 & 0.926 & 0.942 & 1.102 & 0.969 & 0.929 & 0.925 & 0.954 \\
\hline \multicolumn{9}{|l|}{ Validation } \\
\hline MolProbity score & 1.57 & 1.78 & 1.99 & 1.77 & 1.82 & 1.85 & 1.83 & 2.01 \\
\hline Clashscore & 5.9 & 10.9 & 14.51 & 10.63 & 12.31 & 12.96 & 12.37 & 15.24 \\
\hline Poor rotamers (\%) & 0 & 0 & 0 & 0.08 & 0.03 & 0.04 & 0 & 0.03 \\
\hline \multicolumn{9}{|l|}{ Ramachandran plot } \\
\hline Favored (\%) & 96.31 & 96.57 & 95.35 & 96.54 & 96.64 & 96.55 & 96.58 & 95.31 \\
\hline Allowed (\%) & 3.69 & 3.43 & 4.65 & 3.46 & 3.36 & 3.45 & 3.42 & 4.68 \\
\hline Disallowed (\%) & 0 & 0 & 0 & 0 & 0 & 0 & 0 & 0.01 \\
\hline
\end{tabular}


bioRxiv preprint doi: https://doi.org/10.1101/2021.06.29.450292; this version posted June 29, 2021. The copyright holder for this preprint

(which was not certified by peer review) is the author/funder, who has granted bioRxiv a license to display the preprint in perpetuity. It is made available under aCC-BY-NC-ND 4.0 International license.

Table S2. Bacterial strains.

\begin{tabular}{|c|c|c|}
\hline Strain ID & Genotype & Figures \\
\hline MG1655 & $\mathrm{F}-, \lambda-, r p h-1, f n r+$ & \\
\hline SFB018 & MG1655, $\Delta m u k B:: n e o R$ & S4 \\
\hline SFB047 & MG1655, mukB::pheS(T251A, A294G)-hygR, pKW20 Para cas9 lambda-red tet & \\
\hline SFB053 & MG1655, $\triangle$ mukFEB::pheS(T251A, A294G)-hygR, pKW20 Para cas9 lambda-red tet & \\
\hline SFB114 & MG1655, mukB(A304C, N857C, C1118S)-TEV-HaloTag(C61V, C262A)::neoR & $5 B, S 5 C$ \\
\hline SFB115 & MG1655, mukB(G67C, C1118S)-TEV-HaloTag(C61V, C262A)::neoR & $6 \mathrm{C}, \mathrm{S} 5 \mathrm{~B}$ \\
\hline SFB116 & MG1655, mukB(C1118S)-TEV-HaloTag(C61V, C262A)::neoR & $5 B, S 5 B, S 5 C$ \\
\hline SFB117 & MG1655, mukB(A304C, C1118S)-TEV-HaloTag(C61V, C262A)::neoR & 5B \\
\hline SFB118 & MG1655, mukB(N857C, C1118S)-TEV-HaloTag(C61V, C262A)::neoR & 5B \\
\hline SFB119 & MG1655, mukB(R771C, C1118S)-TEV-HaloTag(C61V, C262A)::neoR & $6 \mathrm{C}$ \\
\hline SFB120 & MG1655, mukB(G67C, C1118S, E1407Q)-TEV-HaloTag(C61V, C262A)::neoR & S5B \\
\hline SFB121 & MG1655, mukB(C730S, R771C, C1118S)-TEV-HaloTag(C61V, C262A)::neoR & $6 \mathrm{C}$ \\
\hline SFB122 & MG1655, mukB(G67C, C1118S, D1406A)-TEV-HaloTag(C61V, C262A)::neoR & S5B \\
\hline SFB126 & MG1655, mukB(A304C, N857C, C1118S, S1366R)-TEV-HaloTag(C61V, C262A)::neoR & S5C \\
\hline SFB127 & MG1655, mukB(A304C, N857C, C1118S, D1406A)-TEV-HaloTag(C61V, C262A)::neoR & S5C \\
\hline SFB128 & MG1655, mukB(A304C, N857C, C1118S, E1407Q)-TEV-HaloTag(C61V, C262A)::neoR & S5C \\
\hline SFB167 & MG1655, mukF(D227C) mukE mukB(C1118S)-TEV-HaloTag(C61V, C262A)::neoR & $6 \mathrm{C}$ \\
\hline SFB168 & MG1655, mukB(C1118S, K1246C)-TEV-HaloTag(C61V, C262A)::neoR & $6 \mathrm{C}$ \\
\hline SFB169 & MG1655, mukF(D227C) mukE mukB(C1118S, K1246C)-TEV-HaloTag(C61V, C262A)::neoR & $6 \mathrm{C}$ \\
\hline SFB170 & MG1655, mukF(Q412C) mukE mukB(C1118S)-TEV-HaloTag(C61V, C262A)::neoR & $6 \mathrm{C}$ \\
\hline SFB171 & MG1655, mukF(Q412C) mukE mukB(R143C, C1118S)-TEV-HaloTag(C61V, C262A)::neoR & $6 C$ \\
\hline SFB172 & MG1655, mukF(Q412C) mukE mukB(R143C, R771C, C1118S)-TEV-HaloTag(C61V, C262A)::neoR & $6 \mathrm{D}, 6 \mathrm{C}$ \\
\hline \multirow[t]{2}{*}{ SFB173 } & MG1655, mukF(D227C, Q412C) mukE mukB(R143C, C1118S, K1246C)-TEV-HaloTag(C61V, & $6 \mathrm{D}, 6 \mathrm{C}$ \\
\hline & C262A)::neoR & \\
\hline \multirow[t]{2}{*}{ SFB174 } & MG1655, mukF(D227C, Q412C) mukE mukB(R143C, R771C, C1118S, K1246C)-TEV-HaloTag(C61V, & $6 \mathrm{C}-\mathrm{F}, 6 \mathrm{H}$ \\
\hline & C262A)::neoR & \\
\hline \multirow[t]{2}{*}{ SFB180 } & MG1655, mukF(D227C, Q412C) mukE mukB(R143C, R771C, C1118S, K1246C)::pheS(T251A, A294G)- & \\
\hline & hygR, pKW20 Para cas9 lambda-red tet & \\
\hline SFB183 & MG1655, mukB(R143C, C1118S)-TEV-HaloTag(C61V, C262A)::neoR & $6 \mathrm{C}$ \\
\hline SFB184 & MG1655, mukF(D227C) mukE mukB(R771C, C1118S, K1246C)-TEV-HaloTag(C61V, C262A)::neoR & $6 \mathrm{H}, 6 \mathrm{C}$ \\
\hline
\end{tabular}


bioRxiv preprint doi: https://doi.org/10.1101/2021.06.29.450292; this version posted June 29, 2021. The copvright holder for this preprint (which was not certified by peer review) is the author/funder, who has granted bioRxiv a license to display the preprint in perpetuity. It is made available under aCC-BY-NC-ND 4.0 International license.

\begin{tabular}{|c|c|c|}
\hline SFB188 & MG1655, mukB(G67C, R771C, C1118S)-TEV-HaloTag(C61V, C262A)::neoR & $6 \mathrm{C}, 6 \mathrm{~F}$ \\
\hline SFB190 & $\begin{array}{l}\text { MG1655, mukF(D227C, Q412C) mukE mukB(R143C, R771C, C1118S, K1246C, D1406A)-TEV- } \\
\text { HaloTag(C61V, C262A)::neoR }\end{array}$ & $6 \mathrm{D}, 6 \mathrm{H}$ \\
\hline SFB191 & $\begin{array}{l}\text { MG1655, mukF(D227C, Q412C) mukE mukB(R143C, R771C, C1118S, K1246C, E1407Q)-TEV- } \\
\text { HaloTag(C61V, C262A)::neoR }\end{array}$ & 6D \\
\hline SFB192 & MG1655, mukB(G67C, R771C, C1118S, D1406A)-TEV-HaloTag(C61V, C262A)::neoR & $6 \mathrm{~F}$ \\
\hline SFB193 & MG1655, mukB(G67C, R771C, C1118S, E1407Q)-TEV-HaloTag(C61V, C262A)::neoR & $6 \mathrm{~F}$ \\
\hline SFB202 & $\begin{array}{l}\text { MG1655, } \triangle \text { matP, mukF(D227C, Q412C) mukE mukB(R143C, R771C, C1118S, K1246C)-TEV- } \\
\text { HaloTag }(C 61 V, C 262 A):: n e o R\end{array}$ & $6 \mathrm{H}$ \\
\hline SFB203 & $\begin{array}{l}\text { MG1655, mukF(D227C, Q412C) mukE mukB(G67C, R143C, C1118S, K1246C)-TEV-HaloTag(C61V, } \\
\text { C262A)::neoR }\end{array}$ & $6 \mathrm{C}, 6 \mathrm{E}$ \\
\hline SFB204 & $\begin{array}{l}\text { MG1655, mukF(D227C, Q412C) mukE mukB(G67C, R143C, C1118S, K1246C, D1406A)-TEV- } \\
\text { HaloTag(C61V, C262A)::neoR }\end{array}$ & $6 \mathrm{E}$ \\
\hline SFB205 & $\begin{array}{l}\text { MG1655, mukF(D227C, Q412C) mukE mukB(G67C, R143C, C1118S, K1246C, E1407Q)-TEV- } \\
\text { HaloTag(C61V, C262A)::neoR }\end{array}$ & $6 \mathrm{E}$ \\
\hline SFB206 & MG1655, mukF(D227C, Q412C) mukE mukB(G67C, R143C, C1118S)-TEV-HaloTag(C61V, C262A)::neoR & $6 \mathrm{C}$ \\
\hline SFB207 & MG1655, mukF(D227C, Q412C) mukE mukB(G67C, C1118S, K1246C)-TEV-HaloTag(C61V, C262A)::neoR & $6 \mathrm{C}$ \\
\hline
\end{tabular}


Afonine, P.V., Poon, B.K., Read, R.J., Sobolev, O.V., Terwilliger, T.C., Urzhumtsev, A., and

Badrinarayanan, A., Reyes-Lamothe, R., Uphoff, S., Leake, M.C., and Sherratt, D.J. (2012). In vivo architecture and action of bacterial structural maintenance of chromosome proteins. Science 338, 528-531.

Banigan, E.J., and Mirny, L.A. (2019). Limits of Chromosome Compaction by Loop-Extruding Motors. Phys. Rev. X 9, 031007.

Bürmann, F., Shin, H.-C., Basquin, J., Soh, Y.-M., Giménez-Oya, V., Kim, Y.-G., Oh, B.-H., and Gruber, S. (2013). An asymmetric SMC-kleisin bridge in prokaryotic condensin. Nat Struct Mol Biol 20, 371-379.

Bürmann, F., Lee, B.-G., Than, T., Sinn, L., O’Reilly, F.J., Yatskevich, S., Rappsilber, J., Hu, B., Nasmyth, K., and Löwe, J. (2019). A folded conformation of MukBEF and cohesin. Nat. Struct. Mol. Biol. 26, 227-236. DNA Exit Gate Is Distinct from Its Entrance Gate and Is Regulated by Acetylation. Cell 150, 961-974.

Chapard, C., Jones, R., van Oepen, T., Scheinost, J.C., and Nasmyth, K. (2019). Sister DNA Entrapment between Juxtaposed Smc Heads and Kleisin of the Cohesin Complex. Mol. Cell 75, 224-237.e5.

1092 Collier, J.E., Lee, B.-G., Roig, M.B., Yatskevich, S., Petela, N.J., Metson, J., Voulgaris, M., 1093 Gonzalez Llamazares, A., Löwe, J., and Nasmyth, K.A. (2020). Transport of DNA within cohesin involves clamping on top of engaged heads by Scc2 and entrapment within the ring by Scc3. ELife 9.

Croll, T.I. (2018). ISOLDE: a physically realistic environment for model building into lowresolution electron-density maps. Acta Crystallogr. Sect. Struct. Biol. 74, 519-530.

1098 Cronan, J.E. (2014). The chain-flipping mechanism of ACP (acyl carrier protein)-dependent enzymes appears universal. Biochem. J. 460, 157-163.

1100 Cuylen, S., Metz, J., and Haering, C.H. (2011). Condensin structures chromosomal DNA 1101 through topological links. Nat Struct Mol Biol. 
1102

Danilova, O., Reyes-Lamothe, R., Pinskaya, M., Sherratt, D., and Possoz, C. (2007). MukB colocalizes with the oriC region and is required for organization of the two Escherichia coli chromosome arms into separate cell halves. Mol Microbiol 65, 1485-1492.

Davidson, I.F., and Peters, J.-M. (2021). Genome folding through loop extrusion by SMC complexes. Nat. Rev. Mol. Cell Biol.

Davidson, I.F., Bauer, B., Goetz, D., Tang, W., Wutz, G., and Peters, J.-M. (2019). DNA loop extrusion by human cohesin. Science $366,1338-1345$.

Diebold-Durand, M.-L., Lee, H., Ruiz Avila, L.B., Noh, H., Shin, H.-C., Im, H., Bock, F.P., Bürmann, F., Durand, A., Basfeld, A., et al. (2017). Structure of Full-Length SMC and Rearrangements Required for Chromosome Organization. Mol. Cell 67, 334-347.e5.

Dupaigne, P., Tonthat, N.K., Espéli, O., Whitfill, T., Boccard, F., and Schumacher, M.A. (2012). Molecular basis for a protein-mediated DNA-bridging mechanism that functions in condensation of the E. coli chromosome. Mol. Cell 48, 560-571.

Emsley, P., Lohkamp, B., Scott, W.G., and Cowtan, K. (2010). Features and development of Coot. Acta Crystallogr. D Biol. Crystallogr. 66, 486-501.

Engler, C., Kandzia, R., and Marillonnet, S. (2008). A one pot, one step, precision cloning method with high throughput capability. PLoS One 3, e3647.

Fennell-Fezzie, R., Gradia, S.D., Akey, D., and Berger, J.M. (2005). The MukF subunit of Escherichia coli condensin: architecture and functional relationship to kleisins. EMBO J 24, 1921-1930.

Fisher, G.L.M., Bolla, J.R., Rajasekar, K.V., Mäkelä, J., Baker, R., Zhou, M., Prince, J.P., Stracy, M., Robinson, C.V., Arciszewska, L.K., et al. (2021). Competitive binding of MatP and topoisomerase IV to the MukB dimerization hinge. BioRxiv 2021.03.03.433707.

Fredens, J., Wang, K., de la Torre, D., Funke, L.F.H., Robertson, W.E., Christova, Y., Chia, T., Schmied, W.H., Dunkelmann, D.L., Beránek, V., et al. (2019). Total synthesis of Escherichia coli with a recoded genome. Nature $569,514-518$.

Ganji, M., Shaltiel, I.A., Bisht, S., Kim, E., Kalichava, A., Haering, C.H., and Dekker, C. (2018). Real-time imaging of DNA loop extrusion by condensin. Science 360, 102-105.

Gligoris, T.G., Scheinost, J.C., Bürmann, F., Petela, N., Chan, K.-L., Uluocak, P., Beckouët, F., Gruber, S., Nasmyth, K., and Löwe, J. (2014). Closing the cohesin ring: structure and function of its Smc3-kleisin interface. Science 346, 963-967.

Gloyd, M., Ghirlando, R., and Guarné, A. (2011). The Role of MukE in Assembling a Functional MukBEF Complex. J. Mol. Biol. 412, 578-590.

Gruber, S., and Errington, J. (2009). Recruitment of condensin to replication origin regions by ParB/SpoOJ promotes chromosome segregation in B. subtilis. Cell 137, 685-696. 
1137 Gully, D., Moinier, D., Loiseau, L., and Bouveret, E. (2003). New partners of acyl carrier protein detected in Escherichia coli by tandem affinity purification. FEBS Lett. 548, 90-96.

1139 Haering, C.H., and Gruber, S. (2016). SnapShot: SMC Protein Complexes Part I. Cell 164, 3261140 326.e1.

1141 Haering, C.H., Schoffnegger, D., Nishino, T., Helmhart, W., Nasmyth, K., and Löwe, J. (2004). 1142 Structure and stability of cohesin's Smc1-kleisin interaction. Mol Cell 15, 951-964.

1143 Haering, C.H., Farcas, A.-M., Arumugam, P., Metson, J., and Nasmyth, K. (2008). The cohesin ring concatenates sister DNA molecules. Nature 454, 297-301.

1147 Hassler, M., Shaltiel, I.A., Kschonsak, M., Simon, B., Merkel, F., Thärichen, L., Bailey, H.J., 1148 Macošek, J., Bravo, S., Metz, J., et al. (2019). Structural Basis of an Asymmetric Condensin 1149 ATPase Cycle. Mol. Cell 74, 1175-1188.e9.

1150 Higashi, T.L., Eickhoff, P., Sousa, J.S., Locke, J., Nans, A., Flynn, H.R., Snijders, A.P., 1151 Papageorgiou, G., O'Reilly, N., Chen, Z.A., et al. (2020). A Structure-Based Mechanism for DNA 1152 Entry into the Cohesin Ring. Mol. Cell 79, 917-933.e9.

1153 Hiraga, S., Niki, H., Ogura, T., Ichinose, C., Mori, H., Ezaki, B., and Jaffé, A. (1989). Chromosome partitioning in Escherichia coli: novel mutants producing anucleate cells. J Bacteriol 171, 1496-1505.

1156

1157

1158

1159

1160

1161

1162

1163

1164

1165

1166

1167

1168

1169

1170

Hopfner, K.P., Karcher, A., Shin, D.S., Craig, L., Arthur, L.M., Carney, J.P., and Tainer, J.A. (2000). Structural biology of Rad50 ATPase: ATP-driven conformational control in DNA double-strand break repair and the ABC-ATPase superfamily. Cell 101, 789-800.

Inoue, H., Nojima, H., and Okayama, H. (1990). High efficiency transformation of Escherichia coli with plasmids. Gene 96, 23-28.

Ivanov, D., and Nasmyth, K. (2005). A topological interaction between cohesin rings and a circular minichromosome. Cell 122, 849-860.

Kanno, T., Berta, D.G., and Sjögren, C. (2015). The Smc5/6 Complex Is an ATP-Dependent Intermolecular DNA Linker. Cell Rep. 12, 1471-1482.

Käshammer, L., Saathoff, J.-H., Lammens, K., Gut, F., Bartho, J., Alt, A., Kessler, B., and Hopfner, K.-P. (2019). Mechanism of DNA End Sensing and Processing by the Mre11-Rad50 Complex. Mol. Cell.

Kreamer, N.N.K., Chopra, R., Caughlan, R.E., Fabbro, D., Fang, E., Gee, P., Hunt, I., Li, M., Leon, B.C., Muller, L., et al. (2018). Acylated-acyl carrier protein stabilizes the Pseudomonas aeruginosa WaaP lipopolysaccharide heptose kinase. Sci. Rep. 8, 14124. 
Lee, B.-G., Merkel, F., Allegretti, M., Hassler, M., Cawood, C., Lecomte, L., O’Reilly, F.J., Sinn, L.R., Gutierrez-Escribano, P., Kschonsak, M., et al. (2020). Cryo-EM structures of holo condensin reveal a subunit flip-flop mechanism. Nat. Struct. Mol. Biol. 27, 743-751.

Li, Y., Schoeffler, A.J., Berger, J.M., and Oakley, M.G. (2010). The crystal structure of the hinge domain of the Escherichia coli structural maintenance of chromosomes protein MukB. J Mol Biol 395, 11-19.

Lioy, V.S., Cournac, A., Marbouty, M., Duigou, S., Mozziconacci, J., Espéli, O., Boccard, F., and Koszul, R. (2018). Multiscale Structuring of the E. coli Chromosome by Nucleoid-Associated and Condensin Proteins. Cell.

Lobry, J.R. (1996). Asymmetric substitution patterns in the two DNA strands of bacteria. Mol. Biol. Evol. 13, 660-665.

Mäkelä, J., and Sherratt, D. (2020a). SMC complexes organize the bacterial chromosome by lengthwise compaction. Curr. Genet. 66, 895-899.

Mäkelä, J., and Sherratt, D.J. (2020b). Organization of the Escherichia coli Chromosome by a MukBEF Axial Core. Mol. Cell 78, 250-260.e5.

Mastronarde, D.N. (2005). Automated electron microscope tomography using robust prediction of specimen movements. J. Struct. Biol. 152, 36-51.

Matoba, K., Yamazoe, M., Mayanagi, K., Morikawa, K., and Hiraga, S. (2005). Comparison of MukB homodimer versus MukBEF complex molecular architectures by electron microscopy reveals a higher-order multimerization. Biochem Biophys Res Commun 333, 694-702.

Mercier, R., Petit, M.-A., Schbath, S., Robin, S., El Karoui, M., Boccard, F., and Espéli, O. (2008). The MatP/matS site-specific system organizes the terminus region of the $\mathrm{E}$. coli chromosome into a macrodomain. Cell 135, 475-485.

Minnen, A., Bürmann, F., Wilhelm, L., Anchimiuk, A., Diebold-Durand, M.-L., and Gruber, S. (2016). Control of Smc Coiled Coil Architecture by the ATPase Heads Facilitates Targeting to Chromosomal ParB/parS and Release onto Flanking DNA. Cell Rep.

Miyazaki, K. (2015). Molecular engineering of a PheS counterselection marker for improved operating efficiency in Escherichia coli. BioTechniques 58, 86-88.

Muir, K.W., Kschonsak, M., Li, Y., Metz, J., Haering, C.H., and Panne, D. (2016). Structure of the Pds5-Scc1 Complex and Implications for Cohesin Function. Cell Rep 14, 2116-2126.

Muir, K.W., Li, Y., Weis, F., and Panne, D. (2020). The structure of the cohesin ATPase elucidates the mechanism of SMC-kleisin ring opening. Nat. Struct. Mol. Biol. 27, 233-239.

Murayama, Y., and Uhlmann, F. (2013). Biochemical reconstitution of topological DNA binding by the cohesin ring. Nature. 
1205

1206

1207

1208

1209

1210

1211

1212

1213

1214

1215

1216

1217

1218

1219

1220

1221

1222

1223

1224

1225

1226

1227

1228

1229

1230

1231

1232

1233

1234

1235

1236

1237

1238

Murayama, Y., and UhImann, F. (2015). DNA Entry into and Exit out of the Cohesin Ring by an Interlocking Gate Mechanism. Cell 163, 1628-1640.

Nasmyth, K. (2001). Disseminating the genome: joining, resolving, and separating sister chromatids during mitosis and meiosis. Annu. Rev. Genet. 35, 673-745.

Niki, H., and Yano, K. (2016). In vitro topological loading of bacterial condensin MukB on DNA, preferentially single-stranded DNA rather than double-stranded DNA. Sci Rep 6, 29469.

Niki, H., Imamura, R., Kitaoka, M., Yamanaka, K., Ogura, T., and Hiraga, S. (1992). E.coli MukB protein involved in chromosome partition forms a homodimer with a rod-and-hinge structure having DNA binding and ATP/GTP binding activities. EMBO J 11, 5101-5109.

Nolivos, S., Upton, A.L., Badrinarayanan, A., Müller, J., Zawadzka, K., Wiktor, J., Gill, A., Arciszewska, L., Nicolas, E., and Sherratt, D. (2016). MatP regulates the coordinated action of topoisomerase IV and MukBEF in chromosome segregation. Nat Commun 7, 10466.

Nunez, R.V., Polyhach, Y., Soh, Y.-M., Jeschke, G., and Gruber, S. (2021). Gradual opening of Smc arms in prokaryotic condensin. Cell Rep. 35.

Palecek, J.J., and Gruber, S. (2015). Kite Proteins: a Superfamily of SMC/Kleisin Partners Conserved Across Bacteria, Archaea, and Eukaryotes. Structure 23, 2183-2190.

Pettersen, E.F., Goddard, T.D., Huang, C.C., Meng, E.C., Couch, G.S., Croll, T.I., Morris, J.H., and Ferrin, T.E. (2021). UCSF ChimeraX: Structure visualization for researchers, educators, and developers. Protein Sci. Publ. Protein Soc. 30, 70-82.

Prince, J.P., Bolla, J.R., Fisher, G.L.M., Mäkelä, J., Robinson, C.V., Arciszewska, L.K., and Sherratt, D.J. (2021). Acyl Carrier Protein is essential for MukBEF action in Escherichia coli chromosome organization-segregation. BioRxiv 2021.04.12.439405.

Rajasekar, K.V., Baker, R., Fisher, G.L.M., Bolla, J.R., Mäkelä, J., Tang, M., Zawadzka, K., Koczy, O., Wagner, F., Robinson, C.V., et al. (2019). Dynamic architecture of the Escherichia coli structural maintenance of chromosomes (SMC) complex, MukBEF. Nucleic Acids Res. 47, 9696-9707.

Rawdon, E.J., Dorier, J., Racko, D., Millett, K.C., and Stasiak, A. (2016). How topoisomerase IV can efficiently unknot and decatenate negatively supercoiled DNA molecules without causing their torsional relaxation. Nucleic Acids Res. 44, 4528-4538.

Rohou, A., and Grigorieff, N. (2015). CTFFIND4: Fast and accurate defocus estimation from electron micrographs. J. Struct. Biol. 192, 216-221.

Rosenthal, P.B., and Henderson, R. (2003). Optimal determination of particle orientation, absolute hand, and contrast loss in single-particle electron cryomicroscopy. J. Mol. Biol. 333, 721-745. 
1239

1240

1241

1242

1243

1244

1245

1246

1247

1248

1249

1250

1251

1252

1253

1254

1255

1256

1257

1258

1259

1260

1261

1262

1263

1264

1265

1266

1267

1268

1269

1270

Russo, C.J., and Passmore, L.A. (2014). Electron microscopy: Ultrastable gold substrates for electron cryomicroscopy. Science 346, 1377-1380.

Scheres, S.H.W. (2012). A Bayesian view on cryo-EM structure determination. J. Mol. Biol. 415, 406-418.

Shen, B.-Q., Xu, K., Liu, L., Raab, H., Bhakta, S., Kenrick, M., Parsons-Reponte, K.L., Tien, J., Yu, S.-F., Mai, E., et al. (2012). Conjugation site modulates the in vivo stability and therapeutic activity of antibody-drug conjugates. Nat. Biotechnol. 30, 184-189.

Shi, Z., Gao, H., Bai, X.-C., and Yu, H. (2020). Cryo-EM structure of the human cohesin-NIPBLDNA complex. Science 368, 1454-1459.

Srinivasan, M., Scheinost, J.C., Petela, N.J., Gligoris, T.G., Wissler, M., Ogushi, S., Collier, J.E., Voulgaris, M., Kurze, A., Chan, K.-L., et al. (2018). The Cohesin Ring Uses Its Hinge to Organize DNA Using Non-topological as well as Topological Mechanisms. Cell 173, 1508-1519.e18.

Stoddart, J.F. (2009). The chemistry of the mechanical bond. Chem. Soc. Rev. 38, 1802-1820.

Studier, F.W. (2005). Protein production by auto-induction in high density shaking cultures. Protein Expr Purif 41, 207-234.

Vazquez Nunez, R., Ruiz Avila, L.B., and Gruber, S. (2019). Transient DNA Occupancy of the SMC Interarm Space in Prokaryotic Condensin. Mol. Cell 75, 209-223.e6.

Wagner, T., Merino, F., Stabrin, M., Moriya, T., Antoni, C., Apelbaum, A., Hagel, P., Sitsel, O., Raisch, T., Prumbaum, D., et al. (2019). SPHIRE-crYOLO is a fast and accurate fully automated particle picker for cryo-EM. Commun. Biol. 2, 218.

Wang, K., Fredens, J., Brunner, S.F., Kim, S.H., Chia, T., and Chin, J.W. (2016). Defining synonymous codon compression schemes by genome recoding. Nature 539, 59-64.

Waterhouse, A., Bertoni, M., Bienert, S., Studer, G., Tauriello, G., Gumienny, R., Heer, F.T., de Beer, T.A.P., Rempfer, C., Bordoli, L., et al. (2018). SWISS-MODEL: homology modelling of protein structures and complexes. Nucleic Acids Res. 46, W296-W303.

Weitzel, C.S., Waldman, V.M., Graham, T.A., and Oakley, M.G. (2011). A Repeated Coiled-Coil Interruption in the Escherichiacoli Condensin MukB. J Mol Biol.

Wilhelm, L., and Gruber, S. (2017). A Chromosome Co-Entrapment Assay to Study Topological Protein-DNA Interactions. Methods Mol. Biol. Clifton NJ 1624, 117-126.

Wilhelm, L., Bürmann, F., Minnen, A., Shin, H.-C., Toseland, C.P., Oh, B.-H., and Gruber, S. (2015). SMC condensin entraps chromosomal DNA by an ATP hydrolysis dependent loading mechanism in Bacillus subtilis. Elife 4. 
1271 Woo, J.-S., Lim, J.-H., Shin, H.-C., Suh, M.-K., Ku, B., Lee, K.-H., Joo, K., Robinson, H., Lee, J., 1272 Park, S.-Y., et al. (2009). Structural studies of a bacterial condensin complex reveal ATP1273 dependent disruption of intersubunit interactions. Cell 136, 85-96.

1274 Yatskevich, S., Rhodes, J., and Nasmyth, K. (2019). Organization of Chromosomal DNA by SMC 1275 Complexes. Annu. Rev. Genet.

1276 Yu, D., Ellis, H.M., Lee, E.C., Jenkins, N.A., Copeland, N.G., and Court, D.L. (2000). An efficient 1277 recombination system for chromosome engineering in Escherichia coli. Proc Natl Acad Sci U 1278 A 97, 5978-5983.

1279 Zawadzka, K., Zawadzki, P., Baker, R., Rajasekar, K.V., Wagner, F., Sherratt, D.J., and 1280 Arciszewska, L.K. (2018). MukB ATPases are regulated independently by the N-and C-terminal 1281 domains of MukF kleisin. ELife 7. 